\title{
Digital Maturity Assessment - Bisherige Transformation, Typen digitaler Unternehmen, Branchen- und Ländervergleich
}

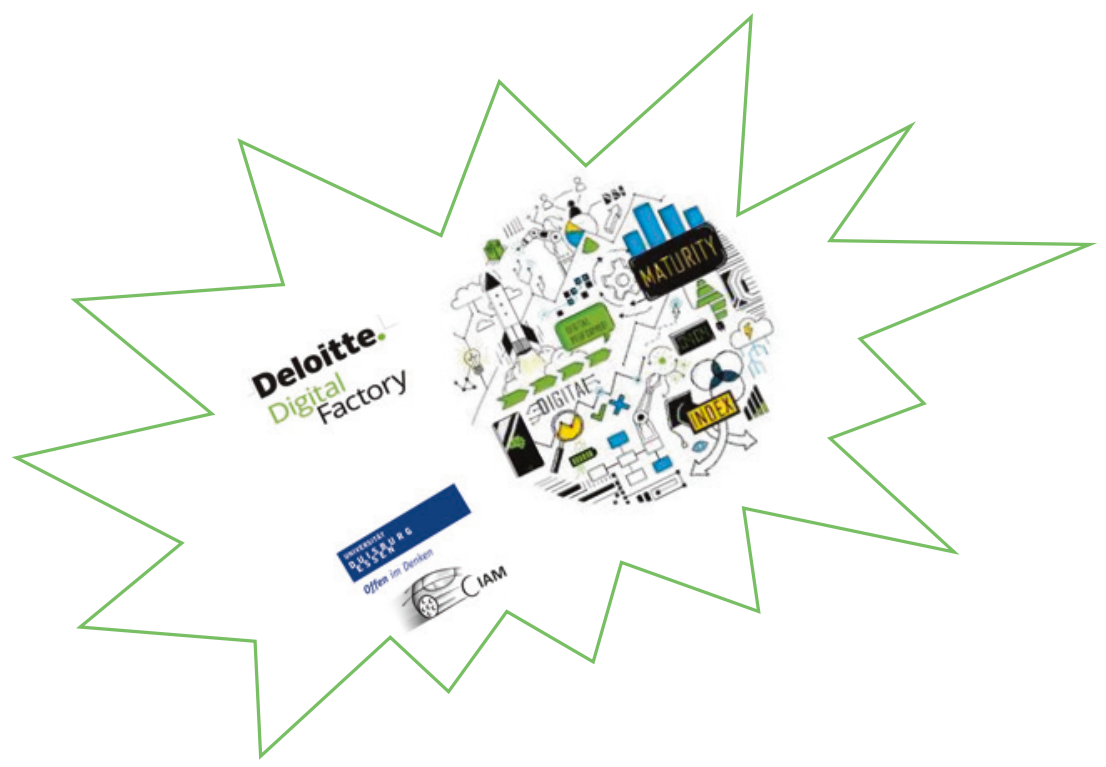


Wir entwickeln in diesem Kapitel einen Index zur Erfassung der digitalen Reife (,Digital Maturity Index“, DMI) und haben damit bei 160 deutschen und 785 Unternehmen weltweit die bisherige digitale Transformation untersucht.

Die Ergebnisse für Deutschland

1. stützen die Hypothesen, dass

- die Gewinnwirkung umso höher ist, je stärker durch die Digitalisierung Technologien und Prozesse (Activities) und mehr noch Angebote und Geschäftsmodelle (Businesses) verändert werden,

- die Gewinnwirkung der Digitalisierung umso höher ist, je stärker Veränderungsfähigkeiten (Dynamic Capabilities) aktiviert und dadurch operative Fähigkeiten (Operational Capabilities) neu konfiguriert werden

- und belegen damit, dass die Gewinnwirkung der Digitalisierung mit der digitalen Reife steigt.

Die Ergebnisse lassen sich entlang der vier Teilindizes (Business Index, Activity Index, Dynamic Capability Index und Operative Capability Index) genauer betrachten.

2. identifizieren sechs Archetypen digitaler Unternehmen (Champions, Potenzialträger, Innovatoren, Optimierer, Folger und Nachzügler) und zeigen, dass nur fünf Prozent der deutschen Unternehmen zu den digitalen Champions zählen, die im Zuge der Digitalisierung sowohl ihre Leistungen als auch ihre Fähigkeiten radikal verändert haben.

Die sechs Typen digitaler Unternehmen unterscheiden sich darin, wie stark sie ihre Leistungen und Kompetenzen, aber auch ihre Wertschöpfung und die IT digitalisieren und wie schnell sie die digitale Transformation managen.

Die Ergebnisse der Unternehmen weltweit

3. ermöglichen einen Branchenvergleich, gemäß dem Anbieter von Industrieprodukten und Dienstleistungsanbieter bereits eine deutlich höhere digitale Reife aufweisen als z. B. Automobilunternehmen.

4. ermöglichen einen Vergleich der bisherigen digitalen Transformation von Unternehmen in der EU, in Amerika und Asien, der relativ große Ähnlichkeiten zwischen den Regionen zeigt. 
In den vorangegangenen Kapiteln wurde gezeigt, dass die Digitalisierung Chancen bietet (Kap. 2), die das Management von Unternehmen nutzen muss, die im Wettbewerb nicht abgehängt werden wollen. Ansatzpunkte dafür bieten Leistungen (Prozesse und Technologien, Angebote und Geschäftsmodelle, Kap. 3), deren Verbesserung und Veränderung digitale Fähigkeiten erfordert (vgl. Kap. 4). Dazu wurden zwei Hypothesen aufgestellt:

1. Die Gewinnwirkung der Digitalisierung eines Unternehmens ist umso höher, je stärker (d. h. radikaler) dadurch Technologien und Prozesse, mehr noch Angebote und am meisten Geschäftsmodelle verändert werden (Kap. 3). Dabei steigt die Radikalität bzw. Diskontinuität der Veränderung von (inkrementell) digitalisierten zu (radikal) digitalen Leistungen (vgl. Kap. 3).

2. Die Gewinnwirkung der Digitalisierung eines Unternehmens ist umso höher, je stärker Veränderungsfähigkeiten (Dynamic Capabilities) aktiviert und dadurch operative Fähigkeiten neu konfiguriert werden (Kap. 4).

Die Hypothesen können zu einer Grundhypothese zur Gewinnwirkung (Profit Impact) der Digitalisierung bzw. der digitalen Reife (Digital Maturity) von Unternehmen zusammengefasst werden (vgl. Abb. 5.1 in Erweiterung von Abb. 3.1 und 4.2): Die Gewinnwirkung der Digitalisierung steigt mit der digitalen Reife.

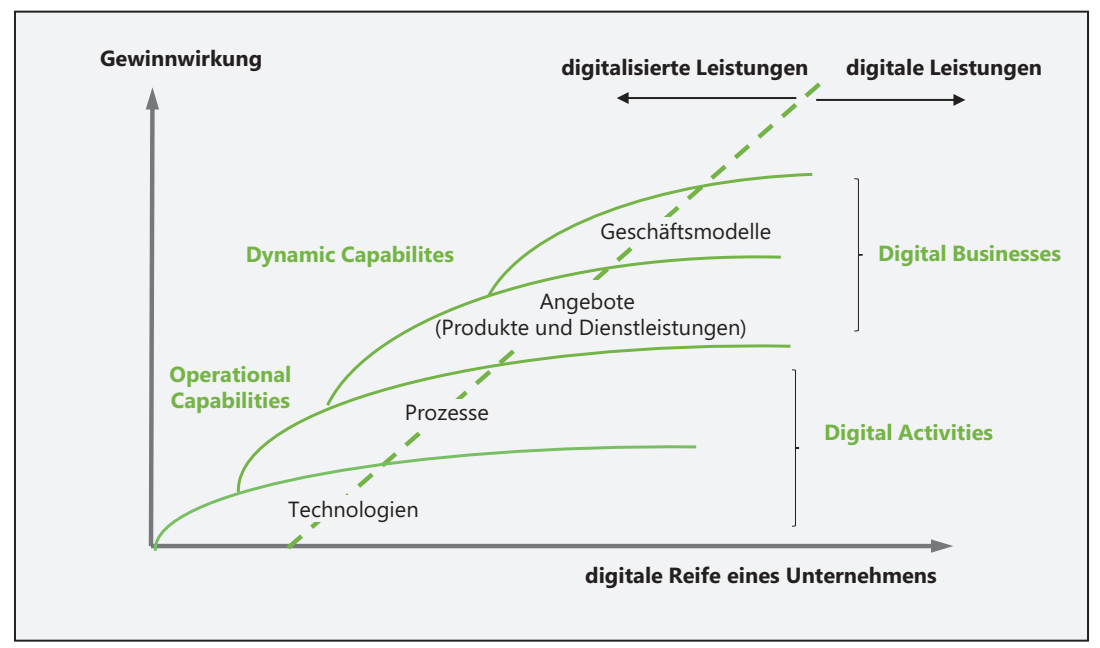

Abb.5.1 Grundhypothese: Positive Korrelation von Gewinnwirkung und digitaler Reife eines Unternehmens. (Quelle: eigener Entwurf in Erweiterung von Abb. 3.1) 
In diesem Kapitel geht es nun darum, über digitale Leistungen und Fähigkeiten in Unternehmen den Status quo und mögliche Potenziale der digitalen Transformation in Deutschland zu erfassen und die Grundhypothese zu prüfen.

Dafür wurde in der Deloitte Digital Factory mit dem Lehrstuhl für Allgemeine Betriebswirtschaftslehre \& Internationales Automobilmanagement der Universität Duisburg-Essen ein Maß der digitalen Reife, der „Digital Maturity Index“ (DMI), entwickelt. Zur Abbildung der verschiedenen Dimensionen des häufig unklar definierten Begriffs „Digitalisierung“ umfasst der Digital Maturity Index entsprechend den Chancen der Digitalisierung in den Kapiteln 3 und 4 vier Teilindizes

- den „Digital Activity Index“, der digitalisierte und digitale Technologien und Prozesse als operative Basis einer Digital Maturity erfasst,

- den „Digital Business Index“, der digitalisierte und digitale (Leistungs-) Angebote und darauf bezogene Geschäftsmodelle erfasst und damit die digitale Wettbewerbsfähigkeit misst,

- den „Dynamic Capability Index“, der Veränderungsfähigkeiten erfasst, die erforderlich werden, um auf Chancen und Risiken der Digitalisierung zu reagieren, sowie

- den „Operational Capability Index“, der individuelle und organisationale Fähigkeiten erfasst, d.h. die operativen Fähigkeiten, die angesichts der Digitalisierung durch die Veränderungsfähigkeiten neu konfiguriert werden müssen.

Der Digital Maturity Index wird hier zunächst vorgestellt (Abschn. 5.1). Er ermöglicht eine Bewertung der digitalen Reife (ein Digital Maturity Assessment) von Unternehmen als Querschnittsbetrachtung zu einem Zeitpunkt und über verschiedene Branchen und (Welt-)Regionen hinweg. Für 160 deutsche Unternehmen werden ein Gesamtindex (Digital Maturity Index) und vier Teilindizes ermittelt, was nicht nur Erkenntnisse über die Digitalisierung ihrer Leistungen und Kompetenzen ermöglicht, sondern auch eine Einschätzung des Ausmaßes der strategischen und operativen Digitalisierung erlaubt (Abschn. 5.2). Mithilfe des Digital Maturity Index lassen sich Archetypen von Unternehmen ${ }^{1}$ unterschiedlicher digitaler Reife unterscheiden und über das Ausmaß der Digitalisierung ihrer Leistungen und Fähigkeiten beschreiben (Abschn. 5.3). Nachdem die Befragung zunächst auf ein Land (Deutschland) begrenzt war, um Ländereffekte auszuschließen, wurde sie später auf 785 Unternehmen weltweit ausgeweitet, um die digitale Reife in einzelnen Branchen (Abschn. 5.4) und in den Regionen Europa, Amerika und Asien (Abschn. 5.5) zu vergleichen. 


\subsection{Digital Maturity Index (DMI): Konzept und Untersuchungsansatz}

Der Digital Maturity Index (DMI) ${ }^{2}$ zeigt den Status quo der digitalen Transformation in einzelnen Unternehmen, einzelner Typen von Unternehmen in Deutschland sowie einzelner Branchen und Regionen weltweit. Er erfasst das Ausmaß der Digitalisierung von Leistungen und Fähigkeiten, durch die sich die Chancen der Digitalisierung nutzen lassen (vgl. die Kap. 3 und 4), d. h. das Ausmaß

1. (inkrementell) digitalisierter oder (radikal) digitaler Technologien und Prozesse (Digital Activities),

2. (inkrementell) digitalisierter oder (radikal) digitaler Angebote (Produkte und Leistungen) und Geschäftsmodelle (Digital Businesses),

3. der Aktivierung von Veränderungsfähigkeiten (Dynamic Capabilities) und

4. der Neu-Konfigurierung von operativen Fähigkeiten (Operational Capabilities).

Entsprechend kann die Digital Maturity über die bereits genannten vier Teilindizes erfasst werden: 1) den Digital Activity Index, 2) den Digital Business Index, 3) den Dynamic Capability Index und 4) den Operational Capability Index (Abb. 5.2).

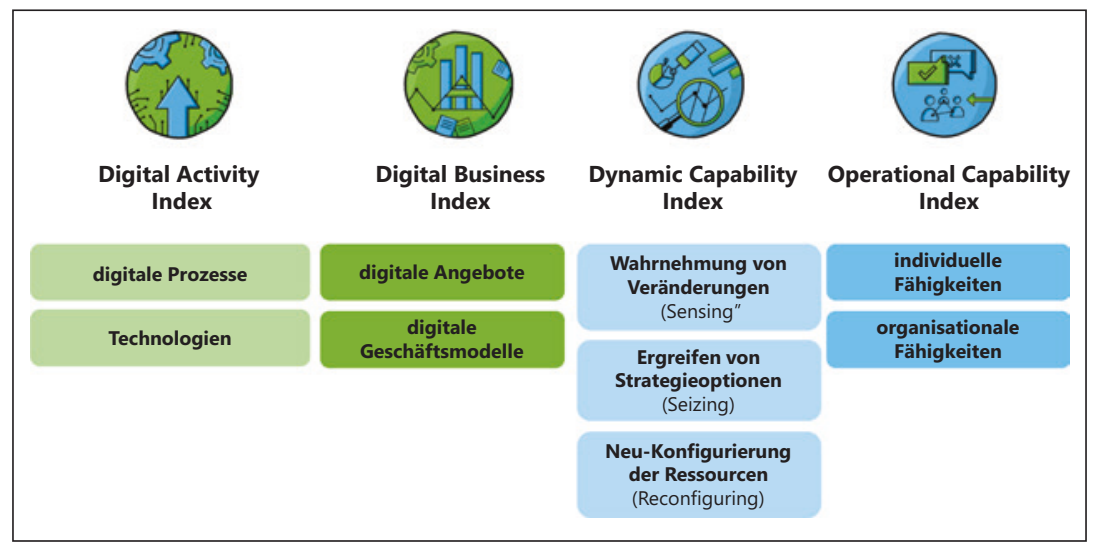

Abb. 5.2 Teilindizes des Digital Maturity Index. (Quelle: eigener Entwurf) 
Weil sich „digitale Reife“ nicht einfach operationalisieren lässt, wurden die Teilindizes als latente Konstrukte $^{3}$ operationalisiert. Jeder der vier Teilindizes wurde über mehrere Items auf einer siebenstufigen Likert-Skala von 1 (keine Digitalisierung) bis 7 (vollständige Digitalisierung) erfasst, wodurch eine metrische Skalierung unterstellt und der Mittelwert der Items berechnet werden kann. Insgesamt wurden 41 Items in allen vier Indizes verwendet. Der Mittelwert der gleichgewichteten Teilindizes ergibt die digitale Reife eines jeden Unternehmens.

\section{Der Digital Activity Index}

Der Digital Activity Index umfasst digitale Prozesse und die zugrunde liegenden digitalen Technologien (vgl. Kap. 3).

Die Digitalisierung der Prozesse und Abläufe kann Verbesserungen oder Veränderungen in allen Funktionsbereichen ermöglichen. Dabei geht es nicht nur um Anpassungen bzw. um eine partielle bzw. inkrementelle Reorganisation einzelner intelligenter (digitalisierter) Prozesse und Gesamtprozesse zur Kostensenkung oder auch Erlössteigerung durch verbesserte Kundeninteraktion, ${ }^{4}$ sondern auch um eine umfassende und tief in die bestehenden Strukturen greifende radikale digitale Neugestaltung der Handlungsspielräume der Unternehmen. Dies ist nur möglich durch neue intelligente Prozesslösungen mittels industrieller Vernetzung in einer smarten Fabrik. ${ }^{5}$ Gerade im Supply Chain Management lässt sich die Effizienz mithilfe von Big Data erheblich steigern. ${ }^{6}$

Diese digitalen Prozesse müssen durch digitale Technologien gestützt werden. Bei einzelnen intelligenten Prozessen hilft ein Manufacturing Execution System (MES) als mehrschichtiges Fertigungsmanagementsystem, das die Führung, Steuerung und Kontrolle der Produktion in Echtzeit u. a. durch Erfassung und Aufbereitung von Betriebs-, Maschinen- und Personaldaten ermöglicht. Aber auch Enterprise Resource Planning (ERP) als komplexe Softwarelösung unterstützt dabei, Ressourcen wie Kapital, Personal, Betriebsmittel, Material und Informationen im Sinne der Unternehmensziele rechtzeitig und bedarfsgerecht zu planen und zu steuern. Intelligente Gesamtprozesse müssen zusätzlich durch ein Product Lifecycle Management (PLM) unterstützt werden, das die Integration aller Informationen ermöglicht, die während des Lebenszyklus eines Produktes anfallen. Ganz neue intelligente Prozesslösungen z. B. in einer smarten Fabrik durch industrielle Vernetzung erfordern neben MES, ERP und PLM umfassende Data Analytics, um Daten aus verschiedenen Quellen zu extrahieren, zu untersuchen und versteckte Muster sowie unbekannte Zusammenhänge zu entdecken.

Im Digital Activity Index wurden insgesamt dreizehn Items zu digitalen Prozessen und Technologien in Anlehnung an Knobbe, Proff (2020) erfasst. Das Ausmaß der Digitalisierung der Prozesse und Technologien wurde mit einer 
7er-Ratingskala von 1 (keine Digitalisierung) bis 7 (vollständige Digitalisierung) erfasst und ungewichtet zu einem Mittelwert für diesen Index zusammengefasst (weil es weder in der Literatur noch in den Gesprächen oder bei den Workshops in der Deloitte Digital Factory Hinweise auf eine Gewichtung gab).

\section{Der Digital Business Index}

Der Digital Business Index bezieht sich auf die Digitalisierung der Angebote Produkte wie Dienstleistungen - und der Geschäftsmodelle (vgl. ebenfalls Kap. 3).

Eine Digitalisierung der Angebote kann gemäß Kap. 3 bereits einfache intelligente Leistungen durch Nutzung intelligenter Infrastruktur $^{7}$ wie z. B. Sensoren bedeuten, die noch auf die traditionelle Leistung bezogen sind und damit einer produkt- bzw. leistungs-dominierten Logik ${ }^{8}$ folgen, die das Produkt, die Dienstleistung oder die Marke ins Zentrum stellt. Weitergehend kann die Nutzung intelligenter Daten über Plattformen intelligente Kundenlösungen schaffen, die einer servicedominierten Logik (,service-dominant logic“9) folgen und die Interaktion zwischen mindestens drei Systemen erfordern, da mindestens zwei Systeme über eine Plattform als einem dritten System kommunizieren.

Eine Digitalisierung der Geschäftsmodelle wurde in Kap. 3 über fünf Komponenten definiert: Ressourcenallokation und Wettbewerbsvorteile als Komponenten von Wettbewerbsstrategien sowie Wertarchitektur, Nutzenversprechen und Gewinnmodell. ${ }^{10}$ Durch die Digitalisierung werden Unternehmen

- entweder ihre bestehenden Geschäftsmodelle verbessern bzw. inkrementell verändern. Dabei zerlegen sie die Wertarchitektur, um Kosten zu senken oder/ und streben in Interaktion mit dem Kunden nach einem individualisierten und integrierten Nutzenversprechen, ${ }^{11}$ um Erlöse zu steigern. Damit verändern sie in digitalisierten Geschäftsmodellen eine oder zwei der leistungsbezogenen Komponenten und dadurch auch die Finanzkomponenten.

- oder sie verändern radikal alle drei leistungsbezogenen Komponenten ihrer Geschäftsmodelle (Wertarchitektur, Nutzenversprechen und Wettbewerbsvorteil) und darüber auch die Finanzkomponenten. Durch eine solche Geschäftsmodellinnovation ${ }^{12}$ entstehen neue digitale Geschäftsmodelle. ${ }^{13}$

Im Digital Business Index wird das Ausmaß der Digitalisierung von Angeboten und Geschäftsmodellen ebenfalls in Anlehnung an Knobbe, Proff (2020) über insgesamt elf Items auf einer 7er-Skala von 1 (keine Digitalisierung) bis 7 (vollständige Digitalisierung) erfasst und zu einem Mittelwert für jedes Unternehmen zusammengefasst. Eine Gewichtung der Items findet wiederum nicht statt, da es in der Literatur und in den Gesprächen und Workshops in der Deloitte Digital Factory keine Hinweise darauf gab. 


\section{Dynamic Capability Index}

Der Dynamic Capability Index erfasst das Ausmaß der Veränderungsfähigkeiten von Unternehmen in Zeiten der Digitalisierung. Er basiert auf einer Operationalisierung von David J. Teece (2007), der Transformationskenntnisse von operativen Fähigkeiten bzw. Kompetenzen unterscheidet. Veränderungsfähigkeiten sind danach definiert als Prozess des Sensing, Seizing und Reconfiguring Abb. (4.1 in Kap. 4). Dabei bedeutet Sensing das Wahrnehmen und Erkennen von Veränderungen und ist die Grundlage für das Seizing als Ergreifen von neuen strategischen Optionen. Reconfiguring sorgt schließlich für die notwendige Kompetenzentwicklung, indem individuelle und organisationale operative Fähigkeiten („ordinary capabilities“) erweitert und neu kombiniert werden. Solche operativen Fähigkeiten sichern den Unternehmensablauf und sind vergleichbar mit Routinen und standardisierten Prozessen im Unternehmen.

Insgesamt wurden im Dynamic Capability Index zwölf Items in Anlehnung an Teece (2007) befragt. Erfasst wurden Tätigkeiten, hinter denen dynamische Fähigkeiten angenommen werden. Die zwölf Werte wurden wiederum zu einem Mittelwert für jedes Unternehmen zusammengefasst. Eine Gewichtung der Fragen findet nicht statt, da es auch dazu in der Literatur keine Hinweise gibt.

\section{Operational Capability Index}

Der Operational Capability Index erfasst schließlich die Digitalisierung operativer (individueller und organisationaler) Fähigkeiten, die durch die Veränderungsfähigkeiten angesteuert werden und die den Wandel implementieren müssen (vgl. Teece 2007). Individuelle Fähigkeiten begründen das vorhandene digitale Know-how sowie Prozesse zum nachhaltigen Aufbau und zur Weiterentwicklung von Wissen. Organisationale Fähigkeiten bewerten das Gestalten von Rollen und Verantwortlichkeiten, die Unterstützung durch das Top-Management sowie die Unternehmenskultur. Zudem wird die Eignung einer Organisation zur Umsetzung einer Digitalisierungs-Roadmap befragt.

Für den Operational Capability Index wurden in Anlehnung an Teece (2018) insgesamt fünf Items erfasst, die analog zum Vorgehen bei den anderen Indizes ungewichtet zu einem Mittelwert pro Unternehmen verdichtet wurden.

Um die Hypothesen zur Gewinnwirkung (den EBIT Impact) der Digital Maturity zu überprüfen, wurde der EBIT Impact als Verbesserung der Profitabilität in sieben gleich großen Abstufungen von 0 bis über 26 Prozent erfasst, und zwar in den sieben EBIT-Intervallen 0, 1 bis 5 Prozent, 6 bis 10 Prozent usw. Diese 
Abstufungen sind die Ergebnisse von Gesprächen und Workshops in der Deloitte Digital Factory in Düsseldorf. Intervalle wurden gewählt, da in einem Pretest mit über 30 Teilnehmern deutlich wurde, dass die Befragten Schwierigkeiten hatten, prozentgenaue Angaben zu machen, und so eher bereit waren, bei diesen doch sensiblen Fragen überhaupt Auskunft zu geben. Als Kontrollvariablen wurden zusätzlich noch der Anteil der zusätzlichen digitalen Leistungen (Umsatzsteigerung) am Gesamtumsatz ebenfalls in sieben Intervallen von 0 bis zu größer 51 Prozent und der Einfluss der Digitalisierung auf die Kostensteigerung in sieben Abstufungen zwischen 0 und größer 21 Prozent erfragt.

Der Pretest der Befragung erfolgte im Oktober/November 2018 bei 30 deutschen Unternehmen, die von Deloitte in Klientenprojekten betreut werden und/ oder wurden, die an Workshops in der Deloitte Digital Factory in Düsseldorf teilnahmen und/oder zu denen der Lehrstuhl für ABWL \& Internationales Automobilmanagement an der Universität Duisburg-Essen Kontakt hält. Die eigentliche Befragung erfolgte von Juni bis Oktober 2019 bei 215 Unternehmen in Deutschland mit über 100 Mio. EUR Umsatz, die zufällig ausgewählt wurden. 160 vollständige Datensätze konnten ausgewertet werden. Für die grundlegende Hypothese zur Gewinnwirkung der Digitalisierung in Abhängigkeit von der digitalen Reife ist diese Stichprobe ausreichend und bietet auch für die weitergehende Unterscheidung von Archetypen eine ausreichend große Fallzahl. ${ }^{14} \mathrm{Da}$ zur Ermittlung von Branchenunterschieden eine größere Stichprobe notwendig war, wurden weitere 928 Unternehmen in der EU, in Nord- und Südamerika sowie in Asien befragt, die ebenfalls zufällig ausgewählt wurden. 785 vollständige Datensätze konnten ausgewählt werden. Die globale Erhebung erfolgte im Oktober und November 2019 als Online-Befragung.

Die Abbildung en 5.3 und 5.4 bieten einige Informationen zu den Stichproben der deutschen und der internationalen Unternehmen. Von den in Deutschland befragten Unternehmen haben 71 Prozent einen Umsatz von mehr als 500 Mio. EUR, 18 Prozent sogar mehr von als 10 Mrd. EUR. 65 Prozent der Studienteilnehmer haben unter 10.000 und 22 Prozent über 20.000 Mitarbeiter. 26 Prozent der Befragten waren auf der Ebene Vorstand/Geschäftsführung (CxO-Level) tätig und 74 Prozent auf einer Führungsebene darunter. Damit stehen die Befragungsteilnehmer recht hoch in den Hierarchien ihrer Unternehmen, weshalb sie einen Überblick über die bisherige digitale Transformation haben dürften. 38 Prozent der in Deutschland Befragten arbeiten in Unternehmen aus dem Industriegütersektor (z. B. Maschinenbau, Konsumgüter, Anlagenbau), 19 Prozent in der Automobilindustrie, 17 Prozent in der Chemie- und Pharmaindustrie und 26 Prozent bei industriellen Dienstleistern (Transport- und Logistik- sowie Ingenieur- und Energiedienstleistern), vgl. Abb. 5.3. 


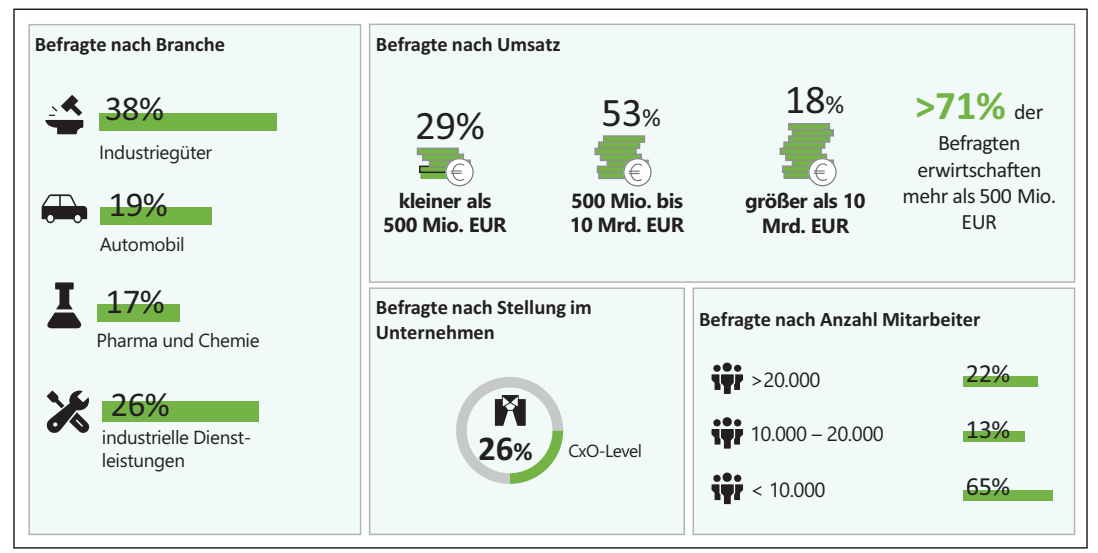

Abb. 5.3 Stichprobe der Untersuchung in Deutschland. (Quelle: eigene Befragung)

Von den 785 befragten ausländischen Unternehmen kommen 43 Prozent aus der EU, 30 Prozent aus Nord- und Südamerika und 27 Prozent aus Asien. Der Anteil der Unternehmen mit einem Umsatz von mehr als 500 Mio. EUR ist höher (79 Prozent) als in der Untersuchung in Deutschland (71 Prozent), gleich groß ist der Anteil der Unternehmen mit einem Umsatz von mehr als $10 \mathrm{Mrd}$. EUR. (18 Prozent). Auch der Beschäftigtenanteil „20.000 und mehr“ ist höher (45 Prozent, vgl. Abb. 5.4). Etwa ein Viertel der Befragten gehört zum Vorstand oder zur Geschäftsführung. Die Branchenverteilung ist etwas anders als in der deutschen Stichprobe: Sehr viel mehr Befragungsteilnehmer arbeiten in Unternehmen aus dem Industriegütersektor (64 statt 38 Prozent), dafür etwas weniger in den anderen Branchen (13 Prozent in der Autoindustrie, 16 Prozent in der Chemieund Pharmaindustrie sowie sieben Prozent bei industriellen Dienstleistern). 


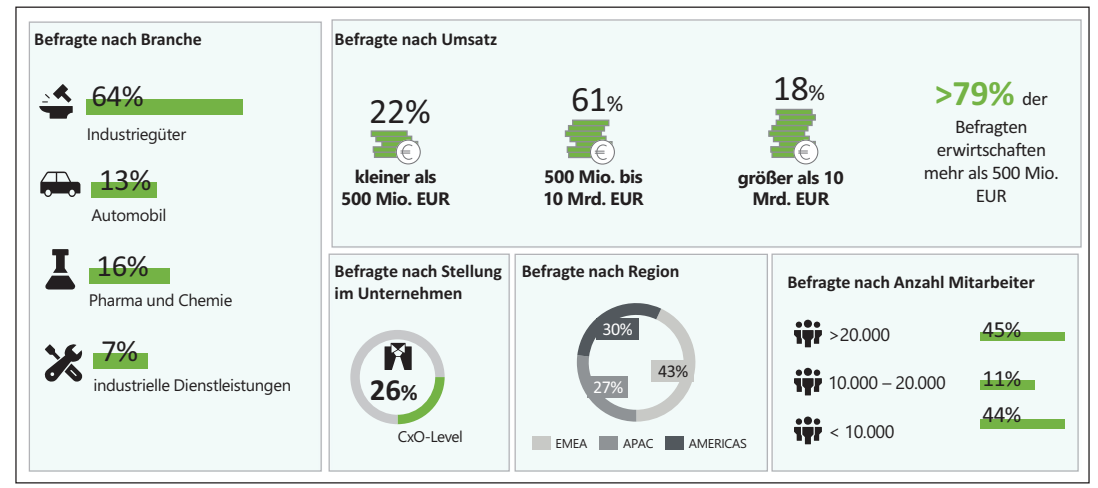

Abb. 5.4 Stichprobe der Untersuchung in der EU, in Amerika und Asien. (Quelle: eigene Befragung)

\subsection{Digital Maturity Assessment (1): Bisherige digitale Transformation deutscher Unternehmen}

Die Befragungsergebnisse belegen die erwartete Korrelation zwischen der digitalen Reife (dem Digital Maturity Index, DMI) und der Gewinnwirkung der Digitalisierung (dem EBIT Impact), vgl. Abb. 5.5. Auch wenn die Ergebnisse dadurch verzerrt werden, dass der EBIT Impact nicht punktgenau, sondern nur in EBIT-Intervallen ( 1 bis 5 Prozent, 6 bis 10 Prozent usw.) angegeben werden konnte, bestätigen die Ergebnisse klar die grundlegende Hypothese der Untersuchung (Abb. 5.1 in Erweiterung von Abb. 3.1 und 4.2), dass die Gewinnwirkung der Digitalisierung mit der digitalen Reife steigt. Wird der Mittelwert der DMIIndexwerte der Unternehmen in einem EBIT-Intervall mit dem mittleren EBIT im EBIT-Intervall korreliert, so ergibt sich ein Korrelationskoeffizient von 96 Prozent, die Güte der linearen Regression ist mit einem $\mathrm{R}^{2}$ von 94 Prozent sehr hoch. Beide Ergebnisse sind hochsignifikant (Signifikanzniveau > 99 Prozent). 


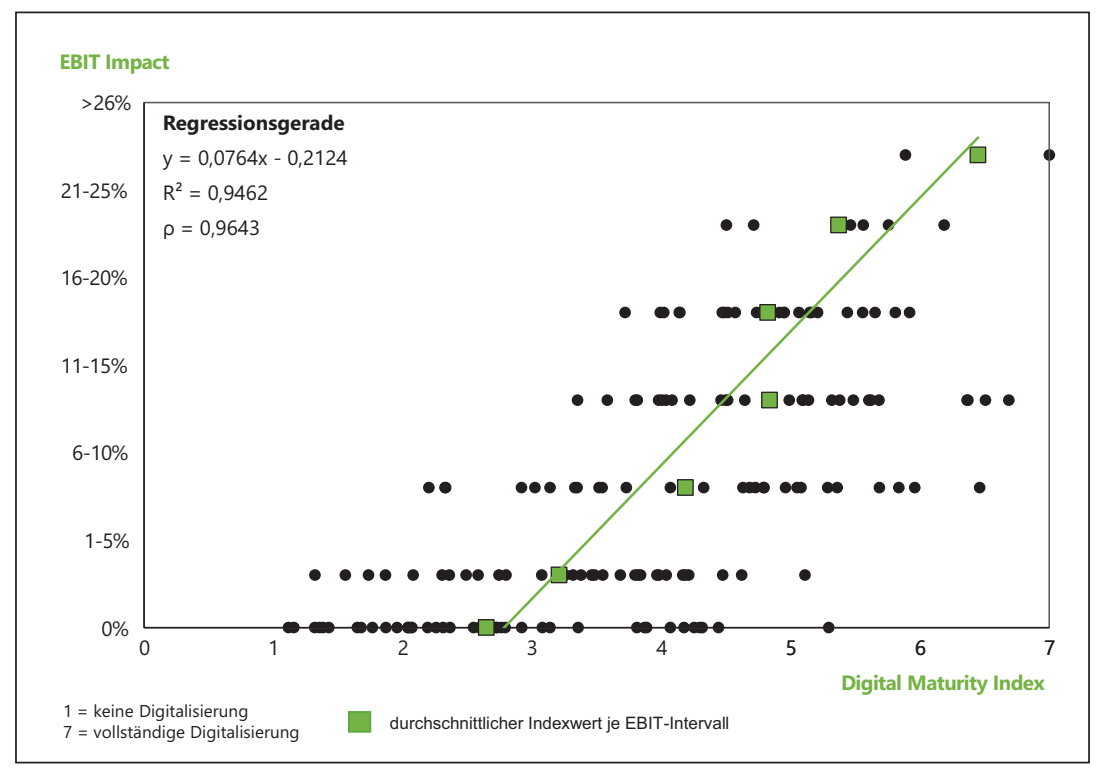

Abb. 5.5 Gewinnwirkung (EBIT Impact) der Digital Maturity. (Quelle: eigener Entwurf)

Die Ergebnisse zeigen, dass die 26 Prozent der befragten Unternehmen, die noch keine Gewinnwirkung sehen, auch ihre digitale Reife am geringsten einschätzen (durchschnittlich 2,75 auf der Skala von 1 (keine Digitalisierung) bis 7 (vollständige Digitalisierung)). Weitere 19 Prozent der befragten Unternehmen, die lediglich eine sehr geringe Gewinnwirkung zwischen einem und fünf Prozent durch die Digitalisierung sehen, bewerten ihre digitale Reife mit durchschnittlich 3,2 ebenfalls gering. Bei den 18, 17 bzw. 14 Prozent der Unternehmen, die eine Gewinnwirkung von zumindest sechs bis 10, 11 bis 15 bzw. sogar 16 bis 20 Prozent angeben, ist die durchschnittlich angegebene digitale Reife bereits deutlich höher (4,4 bzw. zweimal fünf Prozent). Nur fünf bzw. ein Prozent der befragten Unternehmen geben eine hohe Gewinnwirkung der Digitalisierung von 21 bis 25 bzw. sogar über 26 Prozent an, bewerten aber auch ihre digitale Reife am höchsten (mit durchschnittlich 5,3 bzw. sogar 6,5 auf der 7er-Skala).

Überraschend zeigt sich, dass eine Gewinnwirkung erst ab einem Mindestmaß an digitaler Reife (ab einem Mindest-DMI-Indexwert von 2,75 auf der Skala von 1 bis 7) nachgewiesen werden kann. Das bedeutet, dass die 
Digitalisierungsanstrengungen eine kritische Mindesthöhe erreichen müssen, bevor sich ein wirtschaftlicher Effekt einstellt, bzw. dass eine gewisse Bandbreite in den Reaktionen auf die Digitalisierung notwendig ist. Damit begründet sich auch die hohe Unzufriedenheit mit vereinzelten und isolierten digitalen Pilotprojekten, die keine Gewinnwirkung haben können und in den befragten Unternehmen auch nicht haben.

Die Untersuchung zeigt zudem, dass derzeit lediglich sechs Prozent der befragten Unternehmen durch ihre Digitalisierungsaktivitäten eine Gewinnwirkung von über 20 Prozent erreichen und nur 21 Prozent eine Gewinnwirkung von über 15 Prozent sehen. Dagegen erreichen 44 Prozent aller befragten Unternehmen einen EBIT-Impact von maximal fünf Prozent. Diese Ergebnisse bestätigen die Wahrnehmung, dass die Digitalisierung ein hohes Potenzial hat, aber aktuell in Deutschland noch viel zu selten und wenig umgesetzt wird.

Die Untersuchung des DMI bei den 160 deutschen Unternehmen stützt auch die beiden in den Kapiteln 3 und 4 begründeten Einzelhypothesen, dass

1. die Gewinnwirkung der Digitalisierung umso höher ist, je stärker der Grad der Digitalisierung der Leistungen ist, d. h. je stärker (radikaler) dadurch Technologien und Prozesse, mehr noch Angebote und am meisten Geschäftsmodelle verändert werden, und

2. die Gewinnwirkung der Digitalisierung umso höher ist, je stärker digitale Fähigkeiten sind, d. h. je stärker Veränderungsfähigkeiten (Dynamic Capabilities) aktiviert und dadurch operative Fähigkeiten neu konfiguriert werden (vgl. die Abb. 5.6 und 5.7.)

Abb. 5.6 zeigt, dass Unternehmen, die bislang lediglich Technologien und Prozesse für die Digitalisierung eingeführt haben, ohne damit ihre Angebote und Geschäftsmodelle zu digitalisieren, im Durchschnitt eine geringere Gewinnwirkung haben als Unternehmen, die auch ihre Angebote und Geschäftsmodelle digitalisieren. Mit digitalen Prozessen und Technologien ist maximal eine Gewinnwirkung von zehn Prozent möglich, mit digitalen Angeboten jedoch von bis zu 20 Prozent und mit digitalen Geschäftsmodellen sogar von bis zu 26 Prozent. Diese Ergebnisse bestätigen, dass die digitale Transformation in Unternehmen nicht bei der Optimierung von Technologien und Prozessen stehen bleiben darf, um die Chancen der Digitalisierung (vgl. Kap. 2) wirklich zu nutzen. 


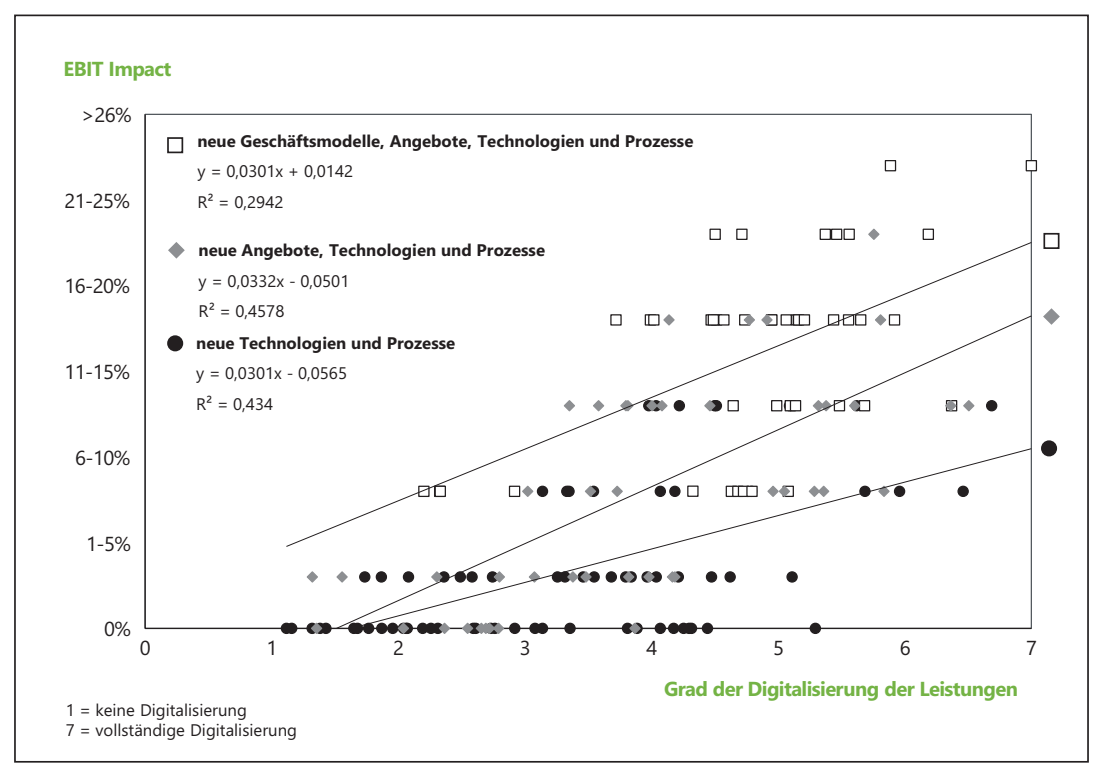

Abb. 5.6 Gewinnwirkungen (EBIT-Impact) der Digitalisierung der Leistungen. (Quelle: eigener Entwurf)

Noch klarer zeigt sich die Bedeutung einer umfassenden Aktivierung von digitalen Fähigkeiten (vgl. Abb. 5.7).

Abb. 5.7 zeigt, dass Unternehmen, die sich bislang darauf beschränken, ihre operativen Fähigkeiten neu zu konfigurieren, im Durchschnitt eine geringere Gewinnwirkung haben als solche, die zunächst Veränderungsfähigkeiten aktivieren, um die Neu-Konfiguration systematisch anzugehen. Alleine mit einer Neu-Konfiguration operativer Fähigkeiten kommen die befragten deutschen Unternehmen nicht über eine 10-Prozentige Gewinnwirkung hinaus, während bei zusätzlicher Aktivierung von Veränderungsfähigkeiten eine Gewinnwirkung von bis zu 26 Prozent erreicht werden konnte. Das zeigt eindeutig, dass Veränderungsfähigkeiten eine wesentliche Voraussetzung für die Nutzung der Chancen der Digitalisierung sind, d. h. die Fähigkeiten, Umfeldveränderungen wahrzunehmen und zu erkennen (Sensing), ggf. neue strategische Optionen (eine Verbesserung oder Veränderung der Prozesse, Angebote und Geschäftsmodelle, vgl. Kap. 3) zu ergreifen (Seizing) und die Kompetenzbasis entsprechend anzupassen (Reconfiguring), vgl. Kap. 4. 


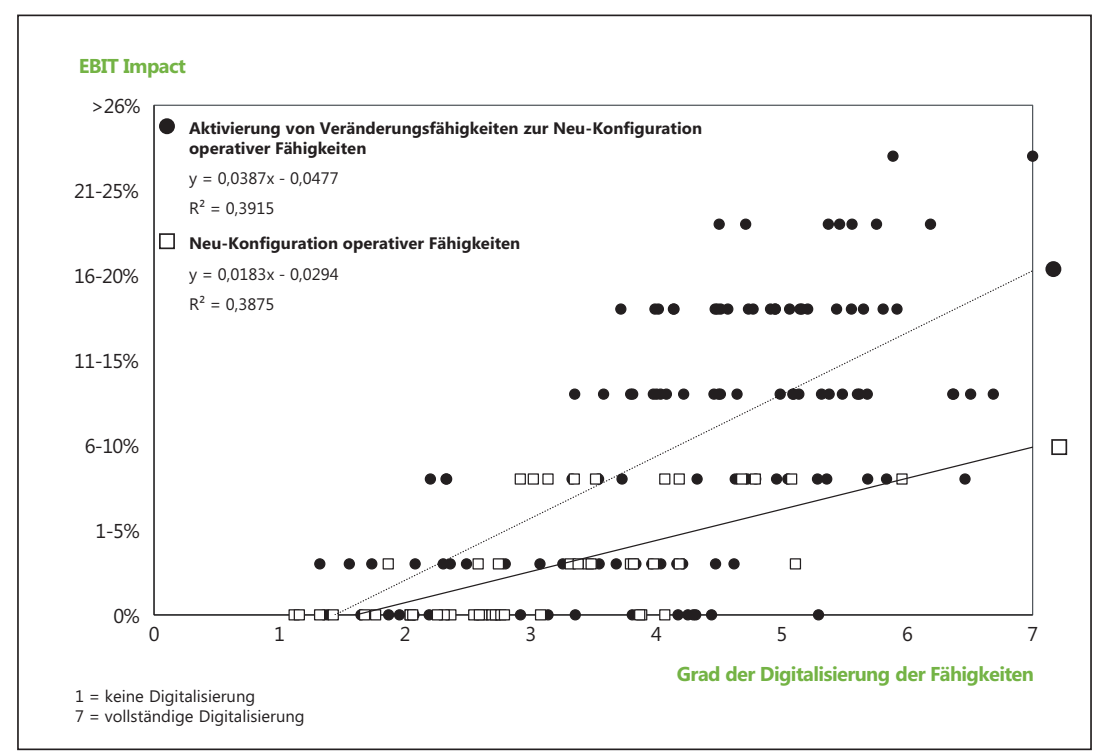

Abb. 5.7 Gewinnwirkungen (EBIT-Impact) der Digitalisierung der Fähigkeiten. (Quelle: eigener Entwurf)

Mithilfe der Teilindizes des Digital Maturity Index lassen sich die Ergebnisse noch präzisieren. Werden z. B.

- die Digitalisierung der Leistungen (Digital Performance), erfasst durch Kombination des Digital Activity Index und des Digital Business Index, und

- die Ausrichtungen der Fähigkeiten auf die Digitalisierung (Digital Capabilities), erfasst durch Kombination des Operational Capability Index und des Dynamic Capability Index,

gegenübergestellt (Abb. 5.8a), so zeigt sich bei den 160 befragten deutschen Unternehmen eine klare Korrelation der Digital Performance und der Digital Capabilities mit einem $\mathrm{R}^{2}$ von 82 Prozent. Damit entwickelt sich die Digitalisierung der Leistungen und der Kompetenzen über die gesamte Stichprobe weitgehend gleichgerichtet: Unternehmen mit einer hohen digitalen Reife der Technologien und Prozesse, Angebote und Geschäftsmodelle haben auch bereits die entsprechenden Kompetenzen aktiviert und umgekehrt. Eine genauere Analyse zeigt, dass die Datenpunkte leicht in Richtung der Leistungen verschoben sind (leichter Versatz der Regressionsgerade mit einem Achsenabschnitt von 0,158 , vgl. Abb. 5.8a). 


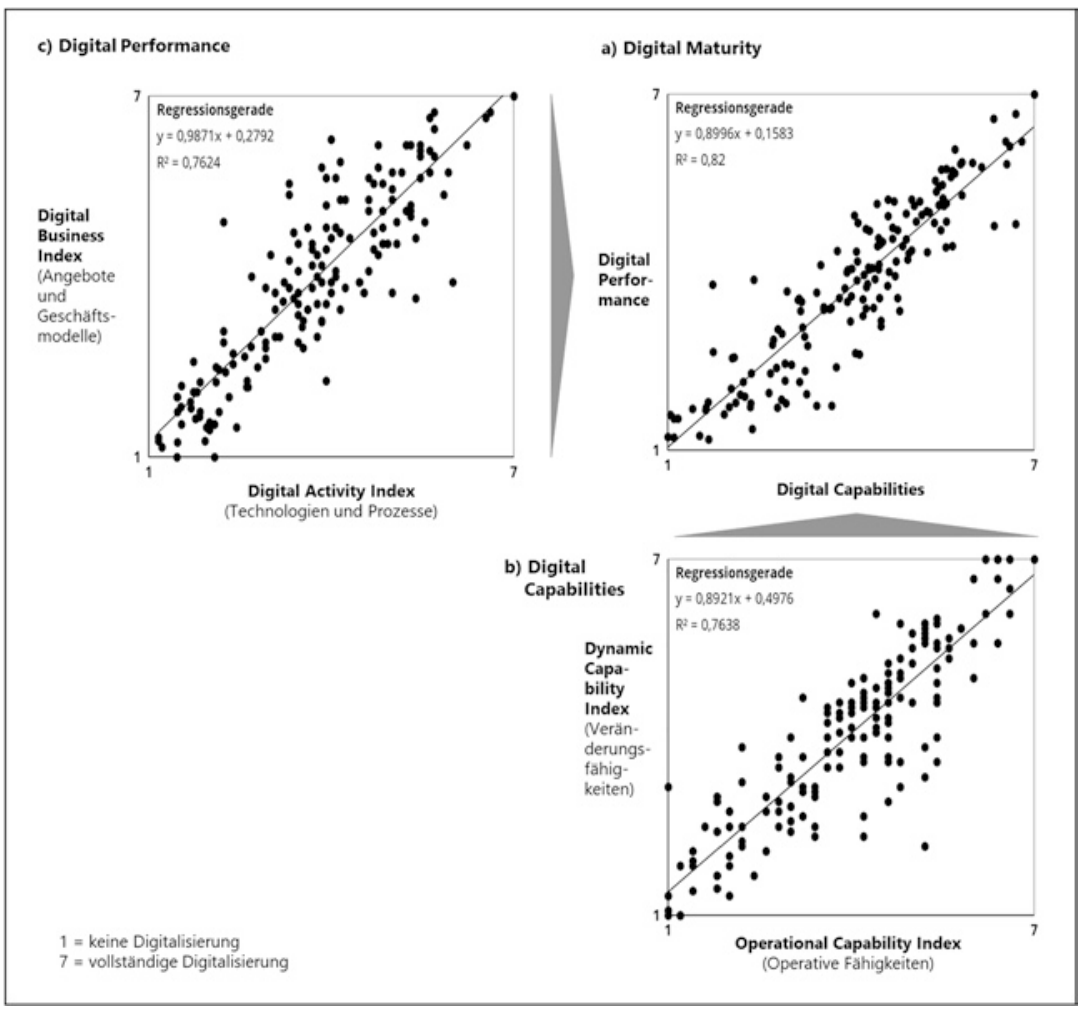

Abb. 5.8 Bislang stärkere Ausrichtung der Fähigkeiten als der Leistungen auf die Digitalisierung. (Quelle: eigener Entwurf)

Dynamische und operative Fähigkeiten sind mit einem $\mathrm{R}^{2}$ von 76 Prozent verknüpft (Abb. 5.8b). Dabei ist die Regressionslinie leicht (Achsenabschnitt von 0,49) in Richtung der Dynamic Capabilities verschoben.

Auch die Gegenüberstellung des Digital Business Index und des Digital Activity Index zeigt deutliche Ausreißer von den Regressionsgeraden $\left(\mathrm{R}^{2}\right.$ von ebenfalls 76 Prozent, vgl. Abb. 5.8c), d. h. eine Verschiebung der Datensätze in Richtung der Digital Businesses (Versatz der Regressionsgeraden mit einem Achsenabschnitt von 0,27) und damit einige Unternehmen, die ihre Angebote und Geschäftsmodelle stärker digitalisieren als ihre Technologien und Prozesse. 
Bei einem anderen Schnitt durch die Teilindizes können zudem

- die Digitalisierung der langfristigeren, strategischen Ausrichtung (Digital Strategy), erfasst als Kombination des Digital Business Index und des Dynamic Capability Index, sowie

- die Digitalisierung der kurzfristigeren, operativen Ausrichtungen, erfasst über den Digital Activity Index und den Operational Capability Index,

gegenübergestellt werden. Dabei zeigt sich für die betrachteten 160 deutschen Unternehmen ebenfalls eine klare Korrelation von strategischer und operativer Ausrichtung auf die Digitalisierung mit einem $\mathrm{R}^{2}$ von 83 Prozent (vgl. Abb. 5.9a).

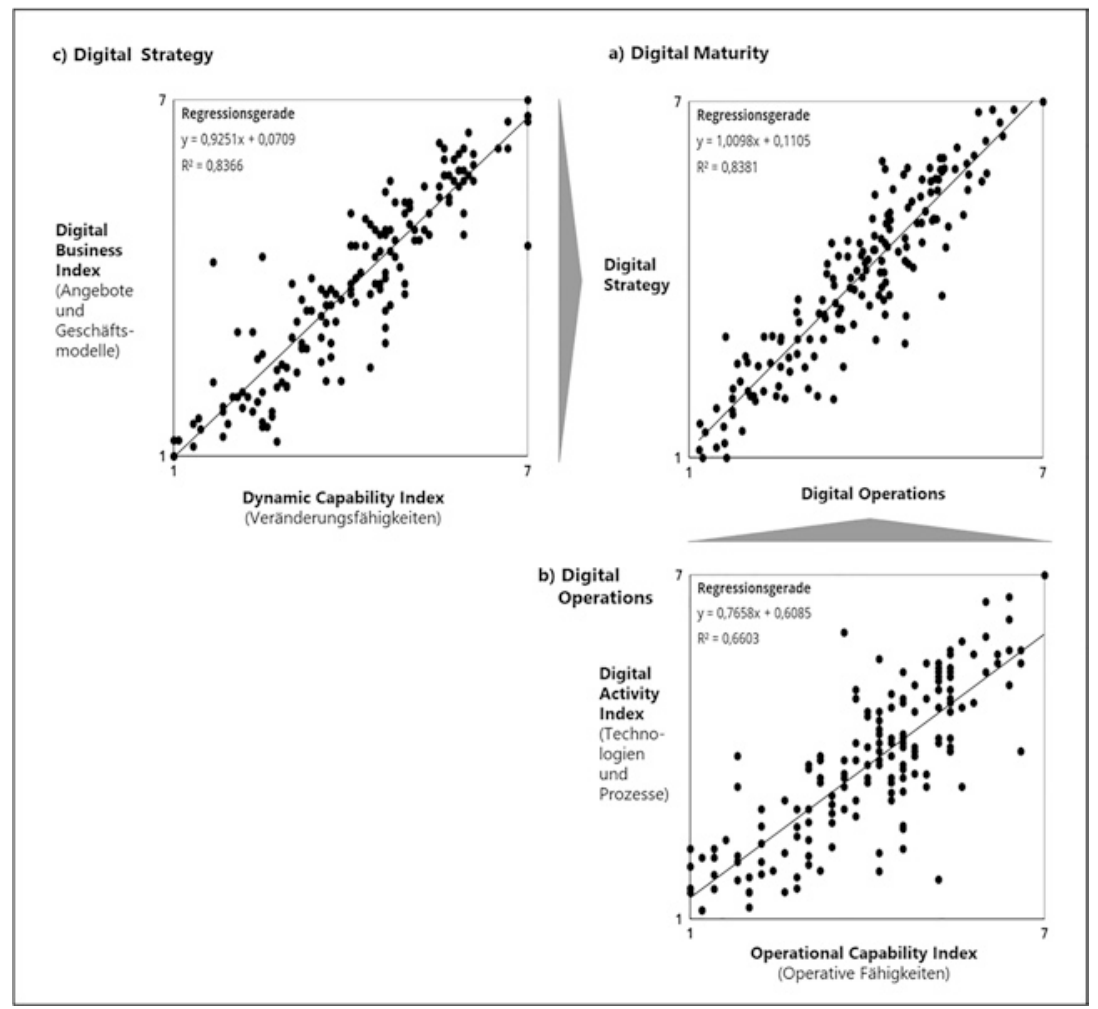

Abb. 5.9 Bislang stärker strategische als operative Ausrichtung auf die Digitalisierung. (Quelle: eigener Entwurf) 
Damit entwickeln sich einerseits die Aktivierung der Veränderungsfähigkeiten sowie die Digitalisierung der Angebote und Geschäftsmodelle und andererseits die Aktivierung der operativen Fähigkeiten sowie die Digitalisierung der Technologien und Prozesse über die gesamte Stichprobe hinweg weitgehend gleichgerichtet. Unternehmen mit einer hohen digitalen strategischen Reife sind auch operativ schon sehr digital aufgestellt und umgekehrt. Es lässt sich aber auch erkennen, dass die befragten deutschen Unternehmen sich bislang etwas stärker strategisch als operativ auf die Digitalisierung ausrichten (Achsenabschnitt der Regressionsgerade bei 0,11).

Während die beiden operativen Indizes der Digital Activities und der Operational Capabilities nur ein geringes $\mathrm{R}^{2}$ von 66 Prozent aufweisen und es viele Ausreißer auf beiden Achsen gibt (vgl. Abb. 5.9b), sind die Digital Businesses und die Dynamic Capabilities mit einem $\mathrm{R}^{2}$ von 83 Prozent enger korreliert (vgl. Abb. 5.9c). Bezüglich der Digital Strategies herrscht bei den 160 befragten deutschen Unternehmen eine höhere Konsistenz als bei den Digital Operations.

Zusammenfassend bewerteten 2019 die 160 teilnehmenden deutschen Unternehmen ihre bisherige digitale Reife im Durchschnitt mit einen DMI-Indexwert von 3,92 auf einer Skala von 1 (keine Digitalisierung) bis 7 (vollständige Digitalisierung). Dies entspricht einer mittleren digitalen Reife und einer noch ausbaufähigen digitalen Transformation. Bei detaillierterer Betrachtung zeigen die verschiedenen Kombinationen der Teilindizes in den Abbildungen 5.8 und 5.9 große Unterschiede in der digitalen Reife der einzelnen Unternehmen. Im nächsten Abschnitt werden deshalb Archetypen digitaler Unternehmen genauer untersucht.

\subsection{Digital Maturity Assessment (2): Archetypen digitaler Unternehmen}

Um die Wege der digitalen Transformation der Unternehmen und somit die zweite (Forschungs-)Frage in Kap. 1 besser zu verstehen, muss die Grundgesamtheit der befragten Unternehmen in Subgruppen aufgeteilt werden. Unternehmen unterscheiden sich in der Interpretation des Handlungsbedarfs und in ihren Veränderungsfähigkeiten auf dem Weg zu einer neuen stabilen Industriestruktur. ${ }^{15}$ Die Ergebnisse wissenschaftlicher Untersuchungen (vgl. Abb. 1.1), aber auch Workshops und Diskussionen in der Deloitte Digital Factory in Düsseldorf haben uns sehr deutlich gezeigt, dass es für die digitale Transformation keinen „One size fits all“-Ansatz gibt. 
Die Abb. 5.8 und 5.9 zeigen eine starke Streuung der Angaben der Unternehmen zu ihrer bisherigen digitalen Reife zwischen 1 (keine Digitalisierung) und 7 (vollständige Digitalisierung) ohne eindeutigen Mittelwert. Deshalb wurde nach verschiedenen Musterbildern (Archetypen) der Digitalisierung in der Stichprobe gesucht. ${ }^{16}$ Eine Clusteranalyse zeigt ähnlich den Ergebnissen in Abb. 5.9 die stabilste Lösung entlang der Diskriminanzachsen „strategische Ausrichtung auf die Digitalisierung“ (Digital Strategy) und „operative Ausrichtung auf die Digitalisierung“ (Digital Operations), vgl. Abb. 5.10. Entlang dieser Achsen lassen sich deutlich sechs Archetypen digitaler Unternehmen unterscheiden - vier davon entlang der 45-Grad-Linie einer gleichmäßigen strategischen und operativen Digitalisierung und jeweils einer ober- bzw. unterhalb dieser 45-Grad Linie. ${ }^{17}$

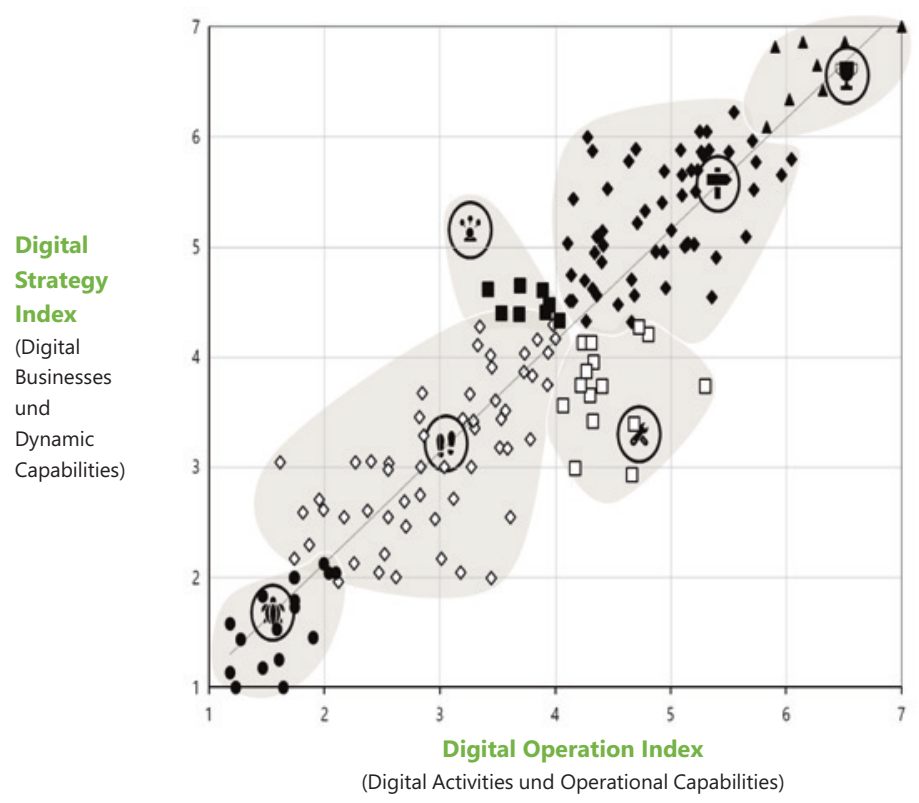

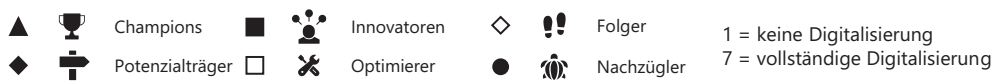

Abb. 5.10 Archetypen digitaler Unternehmen. (Quelle: eigene Darstellung) 
Die Bandbreite der Archetypen reicht von Unternehmen, die sich erst ganz am Anfang ihrer digitalen Reise befinden und oft erst mit einzelnen und isolierten digitalen Pilotprozessen gestartet sind, bis hin zu Unternehmen, die sowohl strategisch als auch operativ bereits nahezu vollständig digitalisiert sind.

In Abhängigkeit vom Ausmaß der strategischen und operativen Digitalisierung lassen sich sechs Archetypen digitaler Unternehmen unterscheiden: (1) digitale Champions, (2) digitale Potenzialträger, (3) digitale Innovatoren, (4) digitale Optimierer, (5) digitale Folger und (6) digitale Nachzügler (vgl. Abb. 5.10). Sie sollen nun kurz vorgestellt und durch die vier Teilindizes des Digital Maturity Index beschrieben werden (Abb. 5.11 bis 5.16).

\section{(1) Digitale Champions}

Fünf Prozent, also acht der befragten 160 deutschen Unternehmen lassen sich dem Archetyp des digitalen Champions zuordnen. Sie haben mit einem durchschnittlichen Digital Maturity Index (DMI) von 6,44 auf der Skala von 1 (keine Digitalisierung) bis 7 (vollständige Digitalisierung, vgl. Abb. 5.11) den höchsten Indexwert in der ganzen Stichprobe. Champions aktivieren nicht nur die Veränderungsfähigkeiten sehr stark (Dynamic Capability Index von 6,74), sondern haben auch bei den anderen Indizes deutlich höhere Werte als die anderen Archetypen. Durch eine etwa gleich starke strategische und operative Ausrichtung auf die Digitalisierung erreichen Champions eine Umsatzsteigerung von durchschnittlich 39 Prozent. Die daraus resultierende Gewinnwirkung liegt bei 16 Prozent und ist damit höher als bei allen anderen Archetypen.

Detaillierter betrachtet haben digitale Champions bereits alle Veränderungsfähigkeiten sehr weitgehend aktiviert, d.h. Fähigkeiten der Wahrnehmung von Umfeldveränderungen (Sensing) wie die Beobachtung und Identifikation von Technologien, Fähigkeiten der strategischen Entscheidungsfindung (Seizing) wie z. B. Strukturierung der Entscheidungen sowie Fähigkeiten der Neu-Konfigurierung der operativen Fähigkeiten (Reconfiguring) z. B. durch Wissensmanagement und Entwicklung von Allianzfähigkeiten (Abb. 5.11a). Gleichzeitig haben sie aber auch ihre Angebote und Geschäftsmodelle schon sehr weitreichend digitalisiert (Digital Business Index von 6,52). In ihrem Angebot geht es weniger um einen hohen Anteil digitaler Leistungen, sondern sie interagieren vor allem über digitale Plattformen und in multilateralen Interaktionen sehr stark mit anderen Systemen und definieren Ecosystemen, um in einem übergeordneten Wertschöpfungsnetzwerk ein unternehmensübergreifendes Nutzenversprechen anzubieten. Gleichzeitig verwenden sie die Digitalisierung für eine Verbesserung des Nutzenversprechens an die Kunden und eine Flexibilisierung der Wertarchitektur sowie dadurch auch für eine Verbesserung von Wettbewerbsvorteilen und Ressourceneinsatz, d. h. für die Veränderung ihrer Geschäftsmodelle (vgl. Abb. 5.11b). 


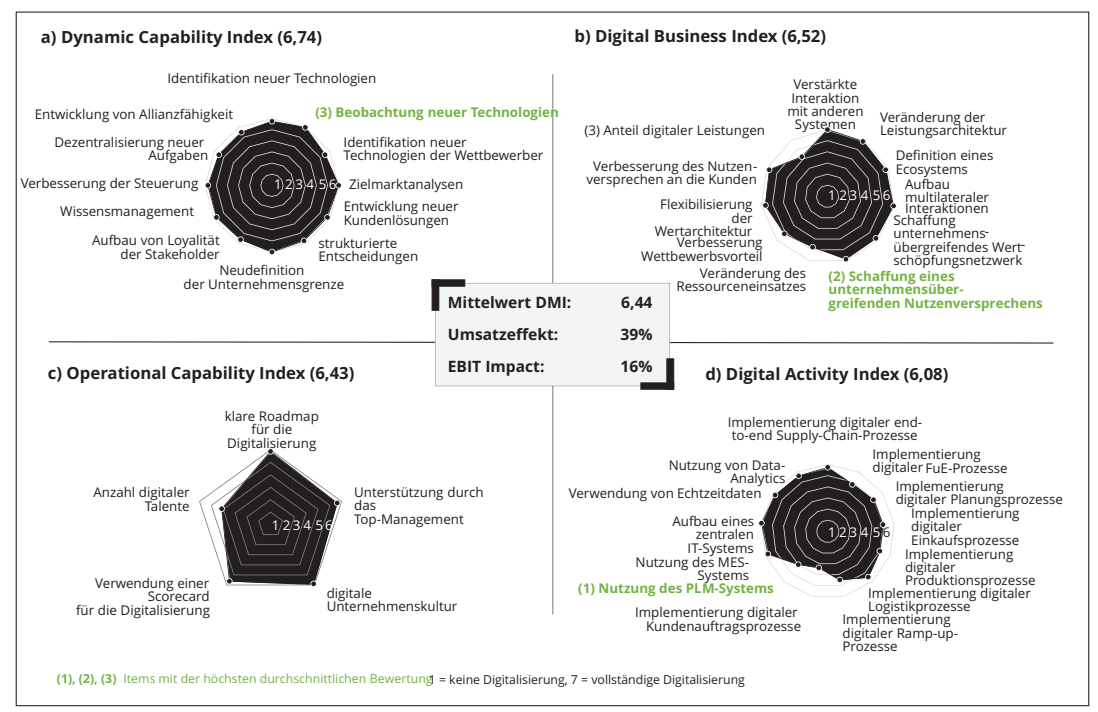

Abb. 5.11 Digital Businesses, Digital Activities, Dynamic Capabilities und Operational Capabilities der digitalen Champions. (Quelle: eigene Darstellung)

Die digitalen Champions sind nicht nur sehr stark bei der strategischen Reaktion auf die Chancen der Digitalisierung, sondern auch bei der operativen Ausrichtung auf die Digitalisierung. Einerseits haben sie ausgeprägte operative Fähigkeiten für die Digitalisierung (Operational Capability Index von 6,43): Sie folgen einer klaren Roadmap bei der Verwendung einer Scorecard für die Digitalisierung und werden durch eine digitale Unternehmenskultur und das Top-Management gestützt (Abb. 5.11c). Andererseits nutzen sie digitale Technologien und Prozesse, legen darauf jedoch nicht den Fokus, denn der Digital Activity Index ist mit ,nur“ 6,08 der von ihnen am wenigsten stark eingeschätzte Teilindex (Abb. 5.11d). Vor allem bei der Nutzung von PLM-Systemen sowie der Implementierung digitaler Kundenauftragsprozesse gibt es noch Potenziale, sie sind hier jedoch trotzdem weiter als die anderen Archetypen digitaler Unternehmen (Abb. 5.12 bis 5.16). 
Über alle vier Teilindizes hinweg bewerten die digitalen Champions (1) die Nutzung von MES-Systemen als Technologien des Fertigungsmanagements, (2) die Schaffung eines unternehmensübergreifenden Nutzenversprechens durch Angebote in einem neuartigen Unternehmensnetzwerk bzw. Ecosystem und (3) die Beobachtung neuer Technologien als dynamische Fähigkeit des Sensing durchschnittlich am höchsten. Dass diese Items zu drei verschiedenen Teilindizes gehören, zeigt die breite digitale Reife der Champions.

\section{(2) Digitale Potenzialträger}

56 der befragten Unternehmen (35 Prozent) ähneln von ihrer digitalen Ausrichtung den Champions, werden aber als digitale Potenzialträger klassifiziert, weil sie die Chancen der Digitalisierung noch nicht ganz so umfassend nutzen wie die Champions. Sie erreichen durchschnittlich einen Digital Maturity Index von 5,08 auf der 7er-Skala (Abb. 5.12). Digitale Potenzialträger aktivieren ihre Fähigkeiten (Dynamic und Operational Capabilities) und digitalisieren gleichermaßen ihre Leistungen, jedoch weniger stark als die digitalen Champions.

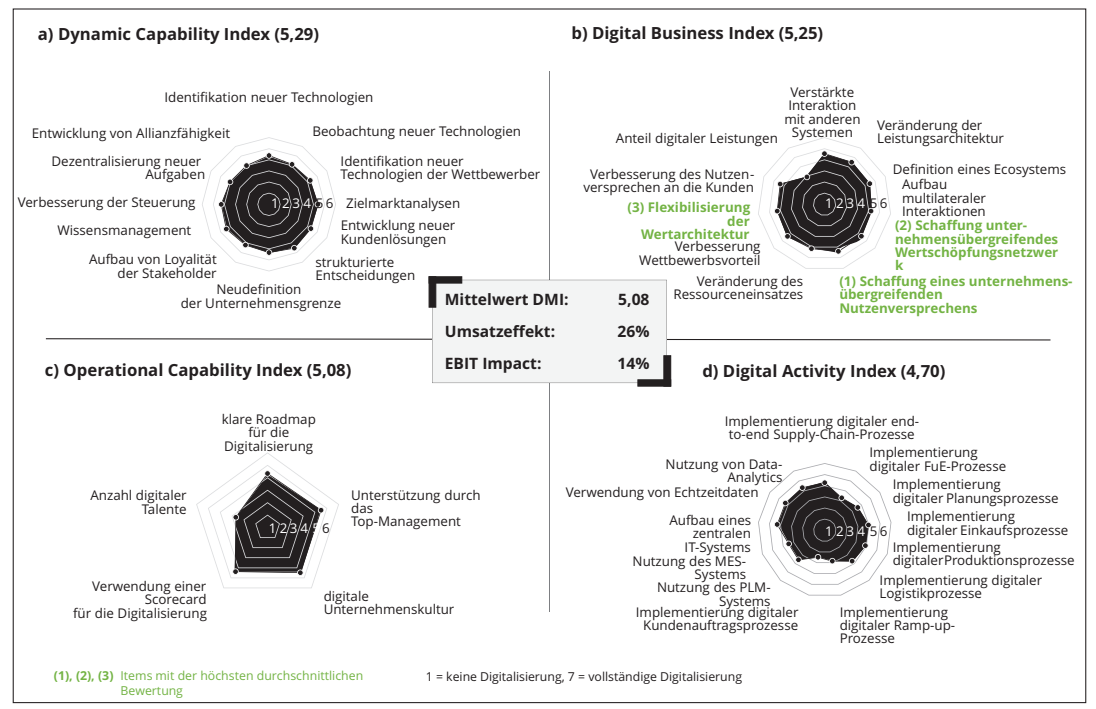

Abb. 5.12 Digital Businesses, Digital Activities, Dynamic Capabilities und Operational Capabilities der digitalen Potenzialträger. (Quelle: eigene Darstellung) 
Bei etwas stärkerer strategischer als operativer Ausrichtung auf die Digitalisierung erreichen sie im Durchschnitt eine Umsatzsteigerung von 26 Prozent und eine Gewinnwirkung von 14 Prozent.

Bei genauerer Betrachtung aktivieren die digitalen Potenzialträger ihre Veränderungsfähigkeiten ähnlich gleichmäßig und mit einem Dynamic Capability Index von 5,29 kaum weniger stark (Abb. 5.12a) als die Champions. Sie digitalisieren auch ihre Angebote und Geschäftsmodelle sehr ähnlich (Digital Business Index von durchschnittlich 5,25), ebenfalls eher durch vernetzte Leistungen in Ecosystemen und neue Geschäftsmodelle als durch einfaches Angebot digitaler Leistungen (Abb. 5.12b). Operativ verändern sie schon recht weitgehend ihre Prozesse, wobei digitale Kundenauftragsprozesse wie bei den Champions noch eher weniger implementiert sind. Noch stärker digitalisieren sie ihre Technologien (Digital Activity Index von insgesamt durchschnittlich 5,08) und nutzen schon weitreichend Data Analytics, verwenden Echtzeitdaten und bauen ein zentrales IT-System auf (Abb. 5.12c). Bei den Operational Capabilities müssen die digitalen Potenzialträger noch etwas nachholen, der entsprechende Index liegt durchschnittlich erst bei 4,70. Vor allem müssen sie die Anzahl ihrer Digital Talents erhöhen (vgl. Abb. 5.12d).

Betrachtet man die vier Teilindizes, bewerten sich die digitalen Potenzialträger im Zuge der Digitalisierung am stärksten (1) bei der Schaffung eines unternehmensübergreifenden Nutzenversprechens (wie die Champions) und (2) bei der Schaffung eines unternehmensübergreifenden Wertschöpfungsnetzwerks in einem neuartigen Unternehmensnetzwerk bzw. Ecosystem sowie (3) bei der Flexibilisierung der Wertarchitektur - einem Element der Veränderung von Geschäftsmodellen - als Voraussetzung für eine Auslagerung und Konzentration auf (neue) Kompetenzen, alles drei Merkmale einer Digitalisierung von Angeboten und Geschäftsmodellen.

Damit ist der digitale Reifegrad der digitalen Potenzialträger zwar noch nicht auf dem Niveau der digitalen Champions, er bewegt sich aber in diese Richtung. Dass zusätzlich zu den fünf Prozent Champions mehr als ein Drittel der befragten Unternehmen zur Gruppe der digitalen Potenzialträger zählt, zeigt, dass in Deutschland schon viele Unternehmen die Zeichen der Zeit erkannt haben und mit der digitalen Transformation schon recht weit gekommen sind.

\section{(3) Digitale Innovatoren}

Fünf Prozent, also wiederum acht der befragten 160 deutschen Unternehmen nutzen die Chancen der Digitalisierung bislang vor allem durch eine strategische Neuausrichtung, ohne die operative Umsetzung gleichermaßen nachzuziehen. Sie 
können als digitale Innovatoren bezeichnet werden. Mit einem Digital Maturity Index von durchschnittlich 4,13 ist zwar der Abstand zu den Potenzialträgern geringer als deren Abstand zu den Champions, aber gerade bei der operativen Ausrichtung auf die Digitalisierung, insbesondere durch Technologien und Prozesse, hinken die digitalen Innovatoren deutlich hinter den Potenzialträgern und Champions her. Sie sind der Archetyp digitaler Unternehmen, bei dem die Unterschiede zwischen den Teilindexwerten am größten sind. Damit erreichen sie im Durchschnitt eine Umsatzsteigerung von 19 und eine Gewinnwirkung von sieben Prozent und bleiben so hinter den digitalen Potenzialträgern und Champions zurück.

Eine genauere Betrachtung der Teilindexwerte der digitalen Innovatoren zeigt, dass sie ihre Veränderungsfähigkeiten mit einem durchschnittlichen Digital Maturity Index von 4,51 schon recht stark aktivieren. Dabei fokussieren sie allerdings bislang noch deutlich stärker auf die Wahrnehmung von Umfeldveränderungen durch die Digitalisierung (Sensing) und die Entscheidungsfindung (Seizing) als auf die Neu-Konfiguration der operativen Fähigkeiten (Reconfiguring), vgl. Abb. 5.13a.

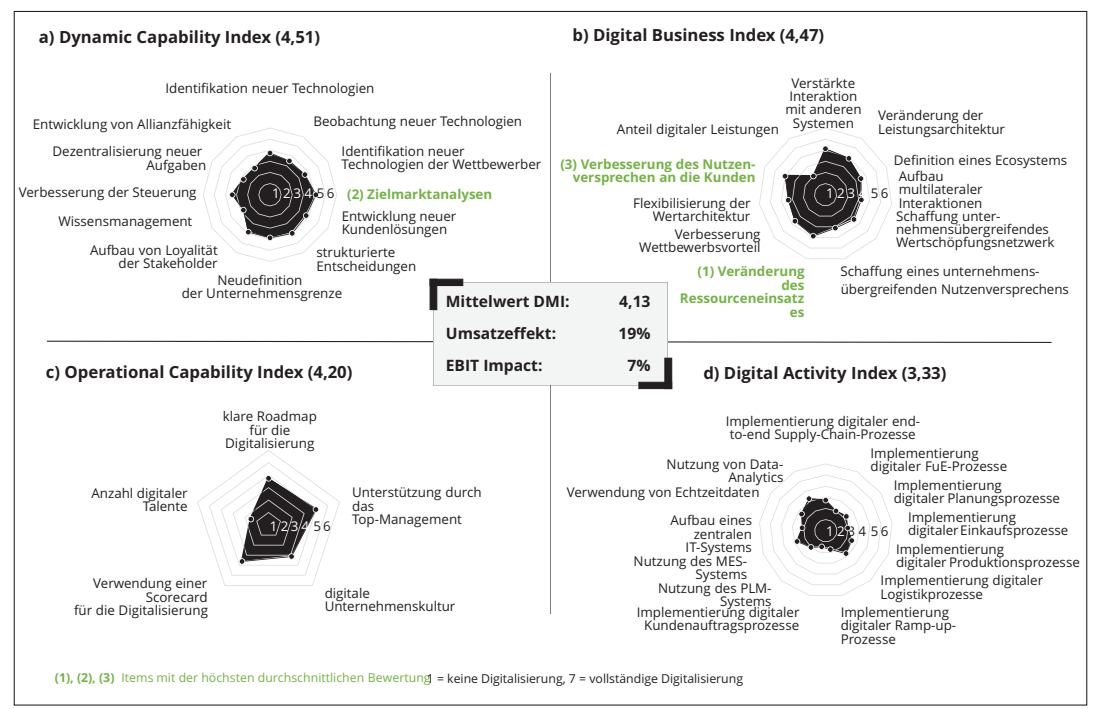

Abb. 5.13 Digital Businesses, Digital Activities, Dynamic Capabilities und Operational Capabilities der digitalen Innovatoren. (Quelle: eigene Darstellung) 
Ihre Angebote und Geschäftsmodelle digitalisieren sie im Durchschnitt ebenfalls bereits recht stark (Digital Business Index von 4,47), allerdings - wie die digitalen Champions und Potenzialträger - mit einem geringen Anteil digitaler Leistungen (Abb. 5.13b). Dass die operative Umsetzung der Digitalisierung bei den Innovatoren noch zurückbleibt, zeigt weniger der Durchschnittswert ihres Operational Capability Index $(4,20)$ als vielmehr der sehr geringe Wert ihres Digital Activity Index (3,33). Für eine weitergehende Digitalisierung der operativen Fähigkeiten fehlen vor allem - und noch stärker als bei den digitalen Potenzialträgern - die entsprechenden Talente und Human Resources (Abb. 5.13c); Technologien und Aktivitäten sind erst ansatzweise digitalisiert (Abb. 5.13d).

Der Blick auf die vier Teilindizes zeigt: Digitale Innovatoren sehen sich im Zuge der Digitalisierung am stärksten (1) bei der Veränderung des Ressourceneinsatzes als Voraussetzung für neue Geschäftsmodelle, (2) bei Zielmarktanalysen im Rahmen der Veränderungsfähigkeit des Sensing (Wahrnehmung der Chancen durch die Digitalisierung) und (3) bei der Verbesserung des Nutzenversprechens an die Kunden als weiterem Element von neuen Geschäftsmodellen, alles drei Merkmale einer strategischen Ausrichtung auf die Digitalisierung.

Damit konzentrieren sich die digitalen Innovatoren bislang vor allem strategisch auf die Chancen der Digitalisierung. Sie aktivieren Fähigkeiten der Wahrnehmung von und der Entscheidung über Veränderungen und digitalisieren daraufhin ihre Angebote sowie Strategien und Geschäftsmodelle. Die operative Umsetzung durch Neu-Konfiguration der operativen Fähigkeiten und die Digitalisierung ihrer Technologien und Prozesse stehen aber noch weitgehend aus. In einem forschungsstarken Land wie Deutschland überrascht der geringe Anteil an Innovatoren.

\section{(4) Digitale Optimierer}

Neun Prozent, das sind 15 der befragten Unternehmen konzentrieren sich bislang auf die Digitalisierung ihrer Technologien und Prozesse, meist in kleinen, inkrementellen Schritten (Abb. 5.14). Entsprechend sind ihre operativen Fähigkeiten stärker als ihre Veränderungsfähigkeiten. Sie können deshalb als digitale Optimierer (der operativen Fähigkeiten und Tätigkeiten) bezeichnet werden. Ihr Digital Maturity Index ist zwar mit durchschnittlich 4,08 ähnlich dem der digitalen Innovatoren $(4,13)$ und deutlich hinter den Werten der digitalen Potenzialträger $(5,08)$ und digitalen Champions $(6,44)$. Mit ihrer klaren operativen Ausrichtung auf die Digitalisierung unterscheiden sie sich jedoch sehr deutlich von den stark strategisch ausgerichteten Innovatoren. 


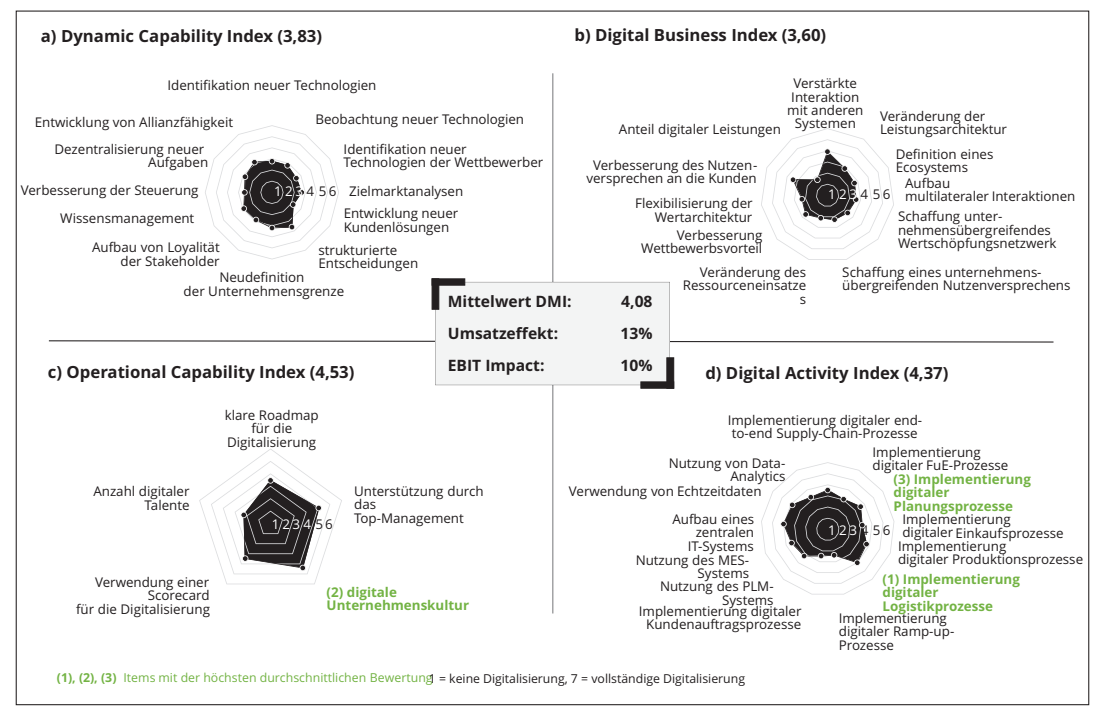

Abb. 5.14 Digital Businesses, Digital Activities, Dynamic Capabilities und Operational Capabilities der digitalen Optimierer. (Quelle: eigene Darstellung)

Weil sie vorrangig ins operative Geschäft investieren, steigt ihr Umsatz durchschnittlich mit 13 Prozent weniger als bei den Innovatoren (19 Prozent), sie erreichen aber bislang eine etwas höhere Gewinnwirkung von zehn Prozent (im Vergleich zu sieben Prozent der Innovatoren).

Eine genauere Betrachtung der Teilindexwerte der digitalen Optimierer belegt ihre noch relativ geringe strategische Ausrichtung auf die Digitalisierung. Sie aktivieren Veränderungsfähigkeiten mit einem durchschnittlichen Digital Maturity Index von 3,83 deutlich weniger als die Innovatoren (4,51), vor allem strategische Entscheidungen über die Entwicklung neuer Kundenlösungen und über die Steuerung der Neu-Konfiguration der operativen Fähigkeiten (Reconfiguring) stehen noch aus (Abb. 5.14a). Deshalb hat die Digitalisierung ihrer Angebote und Geschäftsmodelle auch erst begonnen (Digital Business Index von 3,60, Abb. 5.14b, verglichen mit 4,47 bei den digitalen Innovatoren). Digitale Leistungen z. B. bieten auch sie noch fast gar nicht an. Dafür haben sie aber bereits operative digitale Fähigkeiten entwickelt (Operational Capability Index von 4,53), v. a. gestützt durch die Unternehmenskultur und das Top-Management, trotz einer noch geringen Anzahl digitaler Talente (Abb. 5.14c). Damit konnten sie ihre Technologien und Prozesse schon recht weitreichend digitalisieren, mit einem durchschnittlichen 
Digital Activity Index von 4,37 deutlich stärker als die Innovatoren (3,33). Vor allem durch Technologien der Datensammlung und -nutzung (Data Analytics) und Nutzung von Echtdaten haben sie ein IT-Rückgrat geschaffen und weitreichende digitale Planungs-, Logistik- und Produktionsprozesse implementiert (Abb. 5.14d).

Über alle vier Teilindizes hinweg sehen sich die digitalen Optimierer im Zuge der Digitalisierung entsprechend auch besonders stark bei (1) der Implementierung digitaler Logistikprozesse, (2) bei der digitalen Unternehmenskultur und (3) bei der Implementierung digitaler Planungsprozesse, alles Items der operativen Umsetzung der Chancen der Digitalisierung.

Den digitalen Optimierern mangelt es bislang an einer strategischen Ausrichtung auf die Digitalisierung, insbesondere fehlen innovative Kundenlösungen, aber auch Flexibilität, um auf die Chancen und die Marktdynamik zu reagieren, die die Digitalisierung auslöst. Angesichts der vielen Unternehmen, die darüber klagen, bislang trotz großen Bemühens um Pilotanwendungen und Use Cases noch zu wenig auf die Digitalisierung reagieren zu können, ist die Anzahl der digitalen Optimierer erstaunlich gering. Das ist ein Beleg dafür, dass „hinter den Kulissen“ in vielen Unternehmen schon sehr intensiv an dem Thema Digitalisierung gearbeitet wird.

\section{(5) Digitale Folger}

56 der befragten Unternehmen (35 Prozent) haben sich bislang - strategisch wie operativ - erst wenig auf die Digitalisierung ausgerichtet und bleiben im Durchschnitt (durchschnittlicher Digital Maturity Index von 3,04) und bei allen vier Teilindizes (alle unter 3,3) deutlich hinter den digitalen Innovatoren und Optimierern zurück (Abb. 5.15a bis d). Sie liegen damit nur knapp über der Schwelle, ab der die Digitalisierung gemäß den Ergebnissen in Abb. 5.5 überhaupt eine Gewinnwirkung verursacht, und können als digitale Folger bezeichnet werden. In diesen Unternehmen sind wichtige Entscheidungen zur Digitalisierung häufig noch nicht gefallen, geschweige denn umgesetzt. Deshalb konnten sie bislang auch erst einen Umsatzeffekt von sieben Prozent durch die Digitalisierung erreichen. Die Gewinnwirkung liegt trotz der geringen Umsatzsteigerung zumindest bei fünf Prozent.

Über alle vier Teilindizes hinweg sehen digitale Folger ihre bisherigen Stärken in Bezug auf die Digitalisierung (1) bei der Nutzung von Systemen des Product Lifecycle Managements (PLM), die alle Informationen, die im Verlauf des Lebenszyklus eines Produktes anfallen, integrieren. 


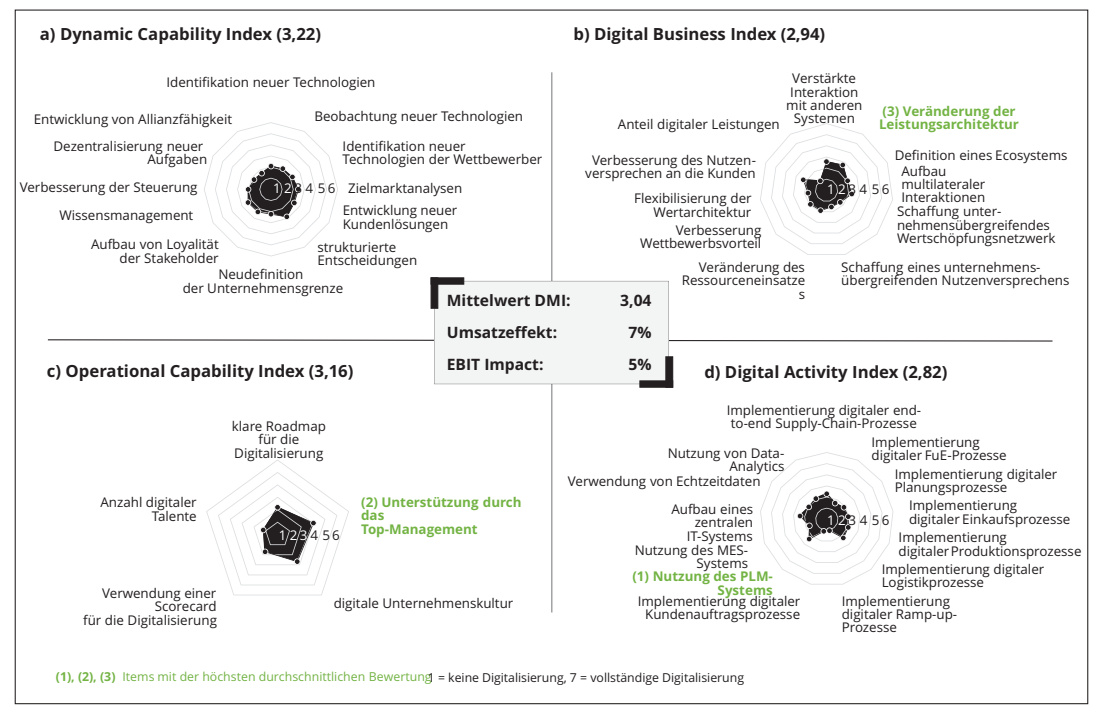

Abb. 5.15 Digital Businesses, Digital Activities, Dynamic Capabilities und Operational Capabilities der digitalen Folger. (Quelle: eigene Darstellung)

(2) betonen sie die große Unterstützung durch das Top-Management und (3) sehen sie bereits Ansätze der Veränderung ihrer Leistungsarchitektur im Zuge der Digitalisierung. Anders als bei den digitalen Champions, bei denen eine solche Streuung der vorrangigen Digitalisierungsbemühungen über Fähigkeiten, Angebote und Technologien als breite Auseinandersetzung mit der Digitalisierung gewertet werden kann, lässt sie bei den digitalen Folgern eher auf ein Verzetteln schließen.

\section{(6) Digitale Nachzügler}

Elf Prozent und damit 17 Unternehmen sind noch kaum digitalisiert (durchschnittlicher Digital Maturity Index von nur 1,61) und können damit als digitale Nachzügler bezeichnet werden. Sie haben die niedrigste Bewertung bei allen vier Teilindizes (zwischen 1,68 und 1,51, Abb. 5.16) und die digitale Transformation bislang häufig erst mit kleineren Pilotprojekten und Initiativen begonnen, die die Verbesserung verschiedener Prozesseffizienzen zum Ziel haben. Weil die Nachzügler damit fast überall unter der Gewinnschwelle bleiben (Abb. 5.5), sind die bisherigen Auswirkungen auf Umsatz und EBIT mit zwei bzw. einem Prozent unbedeutend. 


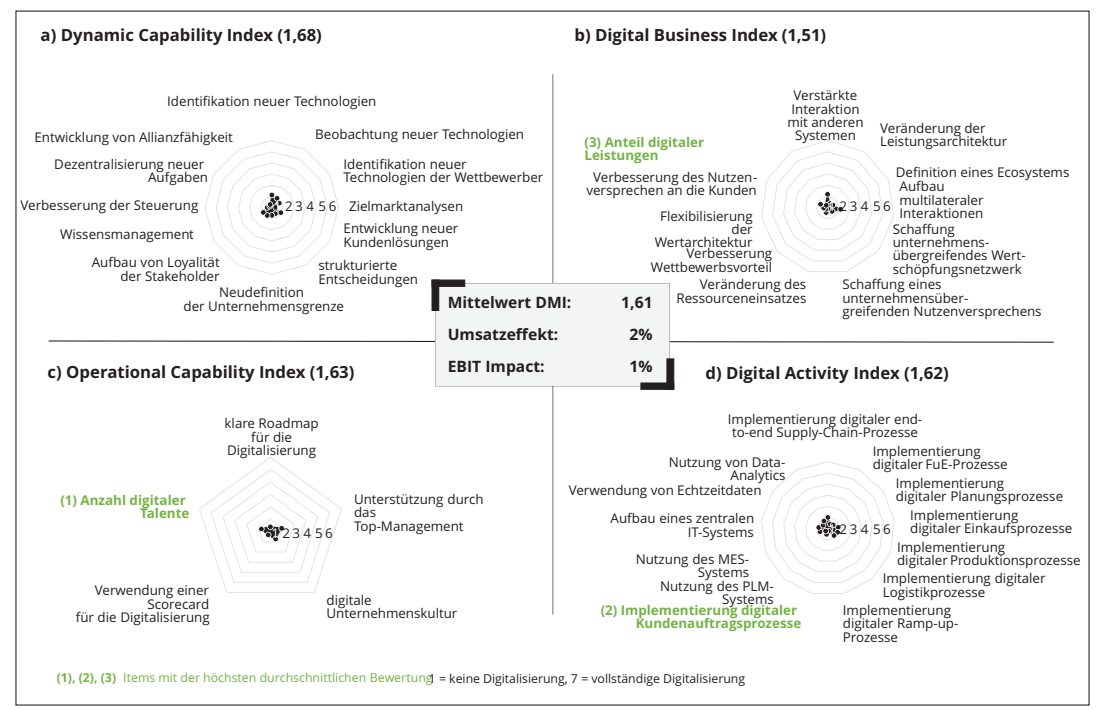

Abb. 5.16 Digital Businesses, Digital Activities, Dynamic Capabilities und Operational Capabilities der digitalen Nachzügler. (Quelle: eigene Darstellung)

In Bezug auf die vier Teilindizes sehen digitale Nachzügler ihre bisherigen Stärken bei der Digitalisierung (1) in der Anzahl digitaler Talente, (2) in der Implementierung digitaler Kundenauftragsprozesse und (3) beim Anteil digitaler Leistungen und damit genau dort, wo die anderen fünf Archetypen digitaler Unternehmen relativ zu anderen Reaktionen auf die Digitalisierung noch eher wenig gemacht haben. Zumindest haben die Nachzügler aber damit begonnen, Mitarbeiter für die digitale Transformation einzustellen und aufzubauen, auch wenn noch ein weiter Weg vor ihnen liegt und die Bedrohung groß ist. Sie kommt nicht nur durch die digitalen Champions, sondern auch durch die digitalen Potenzialträger, Innovatoren und Optimierer, immerhin 54 Prozent der befragten deutschen Unternehmen.

Tab. 5.1 gibt nochmals einen zusammenfassenden Überblick über die durchschnittliche digitale Reife und die mittleren Ausprägungen der einzelnen Teilindizes der sechs Archetypen digitaler Unternehmen. Dabei zeigt sich über alle Archetypen hinweg eine mittelmäßige digitale Reife sowohl beim Gesamtindex (3,92 auf einer Skala von 1 (keine Digitalisierung) bis 7 (vollständige Digitalisierung)) als auch bei allen vier Teilindizes (zwischen 3,68 und 4,08). 
Tab. 5.1 Überblick über die digitale Reife und die Ausprägungen der einzelnen Teilindizes bei den sechs Archetypen digitaler Unternehmen. (Quelle: eigene Darstellung)

\begin{tabular}{|c|c|c|c|c|c|}
\hline $\begin{array}{l}\text { Mittelwerte pro } \\
\text { Archetyp }\end{array}$ & $\begin{array}{c}\text { Dynamic } \\
\text { Capability Index }\end{array}$ & $\begin{array}{l}\text { Digital Business } \\
\text { Index }\end{array}$ & $\begin{array}{c}\text { Operational } \\
\text { Capability Index }\end{array}$ & $\begin{array}{l}\text { Digital Activity } \\
\text { Index }\end{array}$ & Gesamt-DMI \\
\hline Champions & 6,74 & 6,52 & 6,43 & 6,08 & 6,44 \\
\hline Potenzialträger & 5,29 & 5,25 & 5,08 & 4,70 & 5,08 \\
\hline Innovatoren & 4,51 & 4,47 & 4,20 & 3,33 & 4,13 \\
\hline Optimierer & 3,83 & 3,60 & 4,53 & 4,37 & 4,08 \\
\hline Folger & 3,22 & 2,94 & 3,16 & 2,82 & 3,04 \\
\hline Nachzügler & 1,68 & 1,51 & 1,62 & 1,62 & 1,61 \\
\hline gesamte Stichprobe & 4,08 & 3,91 & 4,01 & 3,68 & 3,92 \\
\hline
\end{tabular}

Zwischen den sechs Archetypen bestehen dagegen große Unterschiede im Ausmaß der digitalen Reife: der Digital Maturity Index reicht von 1,61 bei den digitalen Nachzüglern bis 6,44 bei den digitalen Champions.

Tab. 5.1 zeigt auch, dass es zwei klar abgegrenzte Archetypen digitaler Unternehmen am oberen und unteren Rand des Spektrums der digitalen Reife gibt: digitale Champions und digitale Nachzügler, denen zusammen 16 Prozent der befragten 160 deutschen Unternehmen angehören. Dazwischen liegen die vier anderen Archetypen mit Unterschieden im Ausmaß und in den Schwerpunkten ihrer digitalen Transformation. Hier zeigt sich die große Brandbreite möglicher Ansatzpunkte zur Nutzung der Chancen der Digitalisierung, die auch ein Grund dafür ist, dass die Digitalisierung der deutschen Wirtschaft nur langsam vorankommt. Eine Beschleunigung der digitalen Transformation erfordert Fortschritte bei vielen Dimensionen gleichzeitig und eine umfassende funktionsübergreifende Digitalisierung von Kompetenzen, Leistungen und Geschäftsmodellen.

Ein Vergleich der digitalen Reife in Abhängigkeit von der Unternehmensgröße gemessen am Umsatz (Abb. 5.17) zeigt überraschend deutlich einen Größeneffekt. Großunternehmen mit einem Umsatz von über $10 \mathrm{Mrd}$. EUR haben sich insgesamt (mittlerer DMI von 4,40) und bei allen Teilindizes schon stärker auf die Digitalisierung ausgerichtet als mittelgroße Unternehmen mit einem Umsatz von 500 Mio. bis 10 Mrd. EUR (mittlerer DMI von 3,84). Große und mittelgroße Unternehmen haben zudem eine deutlich höhere digitale Reife als kleinere mit einem Umsatz zwischen 100 und 500 Mio. EUR (mittlerer DMI von 2,79). Dieser eindeutige Größeneffekt dürfte daran liegen, dass große Unternehmen eher über die finanziellen Mittel verfügen, um in teure digitale Technologien investieren zu können, und zeigt, dass kleine Unternehmen ihre in der Regel geringere Finanzkraft nicht durch Flexibilität und Schnelligkeit kompensieren können. 


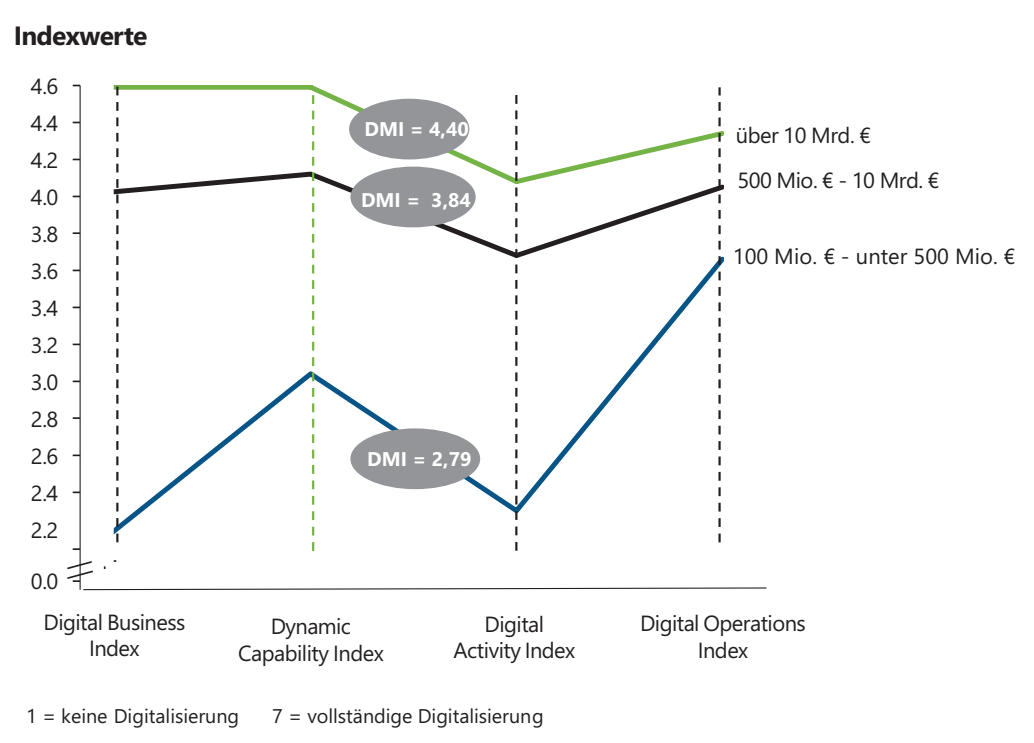

Abb. 5.17 Digitale Reife in Abhängigkeit von der Unternehmensgröße. (Quelle: eigene Darstellung)

\subsection{Digital Maturity Assessment (3): Vergleich der bisherigen digitalen Transformation nach Branchen}

Die Untersuchung der 160 deutschen Unternehmen bietet auch Hinweise auf den Einfluss der Branche, in der ein Unternehmen tätig ist, auf die digitale Reife. In Abschn. 5.1 (Abb. 5.3) wurde die Verteilung der Unternehmen der Stichprobe nach Branchen gezeigt: 38 Prozent sind Anbieter von Industrieprodukten z. B. im Maschinen- oder Anlagenbau $(n=60), 26$ Prozent industrielle Dienstleistungsanbieter (im Ingenieurdienstleistungs- oder Energiesektor und in der Logistik, $\mathrm{n}=42), 17$ Prozent sind Unternehmen der Chemie- und Pharmaindustrie $(\mathrm{n}=27)$ und 19 Prozent Unternehmen der Automobilindustrie $(n=31)$.

Angesichts der geringeren Anzahl Unternehmen in den einzelnen Branchen bei sechs Archetypen ${ }^{18}$ haben wir eine globale Untersuchung bei insgesamt 785 Unternehmen in Europa, Amerika und Asien ergänzt (vgl. ebenfalls Abschn. 5.1, 
Abb. 5.4). Davon sind 64 Prozent $(n=500)$ Unternehmen im Industriegütersektor, sieben Prozent $(n=58)$ industrielle Dienstleister, 16 Prozent $(n=124)$ Unternehmen der Chemie- und Pharmaindustrie und 13 Prozent $(n=103)$ Unternehmen der Automobilindustrie.

Der Vergleich der sechs Archetypen digitaler - deutscher und ausländischer Unternehmen in den vier Branchen (Abb. 5.18) zeigt eine sehr hohe Übereinstimmung der Ergebnisse (vgl. ebenfalls Abb. 5.18): Es gibt in allen vier Branchen wenige digitale Champions und Nachzügler, viele digitale Potenzialträger und Folger und wiederum weniger digitale Innovatoren und Optimierer. Auffällig sind jedoch

1. die höhere digitale Reife der industriellen Dienstleister (mehr Champions, keine Nachzügler, zumindest bei den ausländischen Unternehmen), was darauf zurückzuführen sein dürfte, dass sie aufgrund der geringeren Fixkosten schneller und flexibler sind, und

2. der Rückstand vor allem der deutschen Automobilunternehmen (deutlich weniger digitale Potenzialträger und viele Folger), was daran liegen dürfte, dass sie ihre Kräfte gleichzeitig auf den Übergang in die Elektromobilität und in die digitale Transformation hin zum autonomen Fahren sowie zu plattformbasierten Mobilitätsangeboten aufteilen müssen und dabei nur zögerlich vorangehen.

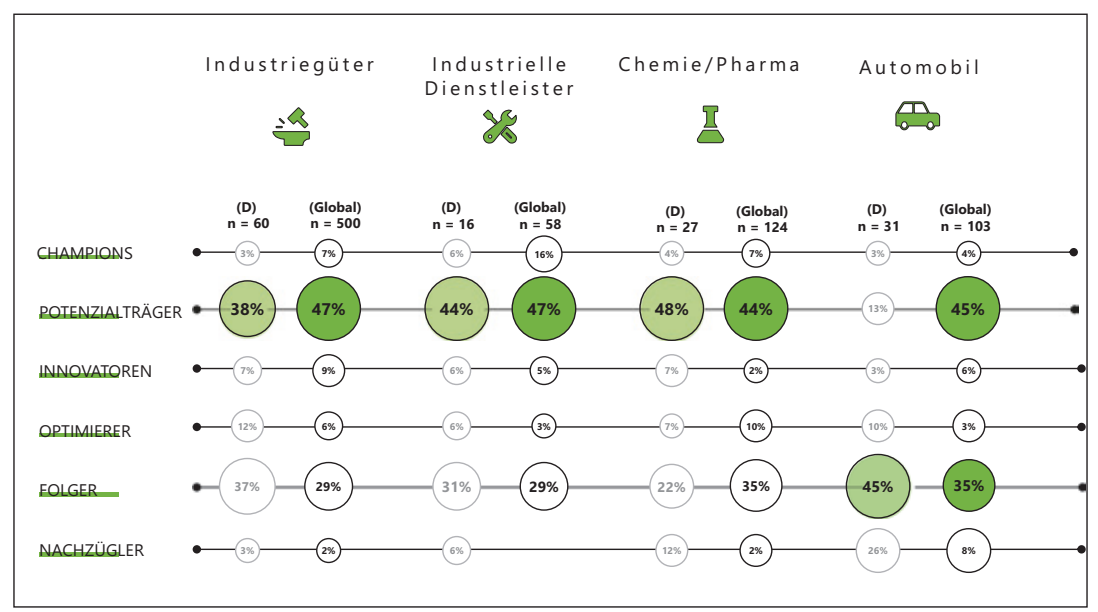

Abb. 5.18 Vergleich der Verteilung der Archetypen digitaler Unternehmen in verschiedenen Branchen in Deutschland und in einer globalen Stichprobe. (Quelle: eigene Darstellung) 


\subsection{Digital Maturity Assessment (4): Vergleich der bisherigen digitalen Transformation in verschiedenen Regionen}

Ein Vergleich der 785 im Ausland befragten Unternehmen bietet schließlich auch Hinweise auf den Einfluss der Region, in der ein Unternehmen tätig ist. In Abschn. 5.1 wurde bereits die Verteilung nach Branchen gezeigt: 339 der Unternehmen kommen aus der EU (ohne Deutschland), 237 aus Amerika und 209 aus Asien (vgl. Abb. 5.4).

Der Vergleich der Regionen zeigt zunächst, dass sich Deutschland und die restlichen EU-Länder bezüglich der digitalen Reife kaum unterscheiden, wahrscheinlich deshalb, weil sie einem regionalen Integrationsraum angehören. Ähnlich wie zwischen Branchen (Abschn. 5.4) gibt es auch zwischen den Regionen EU, Amerika und Asien kaum Unterschiede in der digitalen Transformation (Abb. 5.19). Die Zuordnung der Unternehmen zu den sechs Archetypen ist ebenfalls ähnlich: Es gibt in allen Regionen relativ wenige digitale Champions und Nachzügler, aber auch digitaleInnovatoren und Optimierer, während digitale Folger und Potenzialträger zusammen zwischen 70 und 80 Prozent aller Unternehmen ausmachen. Auffällig ist jedoch im Vergleich zwischen Deutschland und den Regionen Amerika und Asien,

1. dass Amerika mehr als doppelt so viele digitale Champions (elf Prozent) hat wie Deutschland, was daran liegen dürfte, dass viele Basistechnologien der Digitalisierung von großen amerikanischen Technologieunternehmen und ihren Zulieferern kommen, und

2. dass in Asien die Anzahl der digitalen Nachzügler deutlich geringer ist (nur ein Prozent aller Unternehmen) als in Deutschland (elf Prozent), was auf die höhere Technologieaffinität in Asien zurückgeführt werden könnte. Dabei überrascht ein wenig, dass es in Asien nicht auch mehr Champions gibt.

Innerhalb der Regionen (z. B. in Amerika zwischen den USA, Kanada und Brasilien) gibt es zwar auch Unterschiede der digitalen Reife der Unternehmen, eine weitergehende Untersuchung würde aber den Rahmen dieses Buches sprengen, das schwerpunktmäßig die digitale Transformation deutscher Unternehmen betrachtet. 


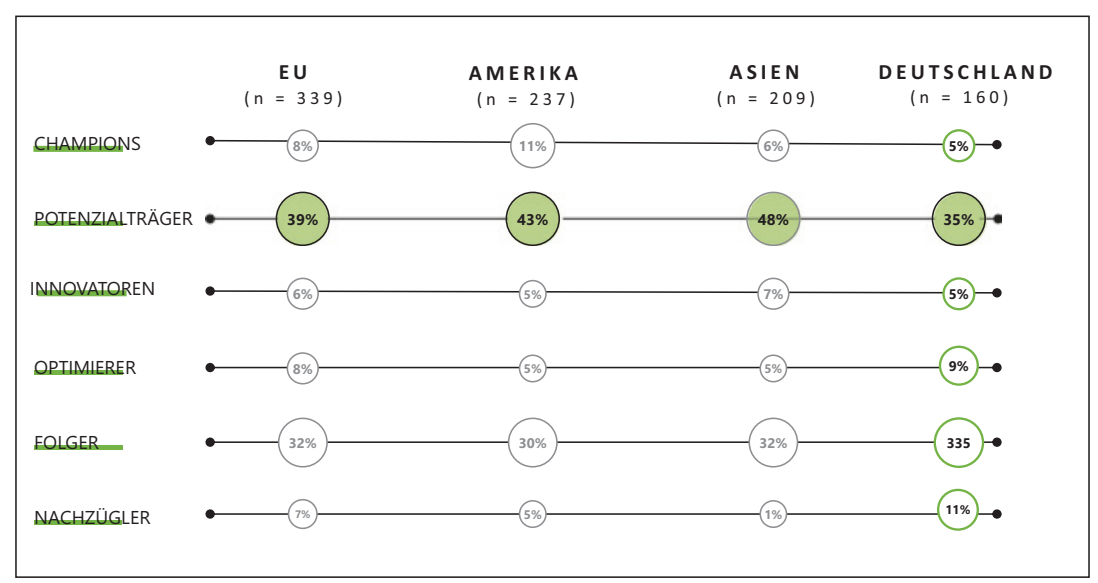

Abb. 5.19 Vergleich der Verteilung der Archetypen digitaler Unternehmen in verschiedenen Regionen. (Quelle: eigene Darstellung)

In diesem Kapitel konnte mittels einer Befragung von 160 deutschen Unternehmen gezeigt werden, dass sie mit der digitalen Transformation begonnen haben, bei einer durchschnittlichen digitalen Reife von 3,92 auf einer 7er-Skala aber noch Verbesserungspotenziale bestehen. Bei detaillierterer Betrachtung zeigen sich allerdings große Unterschiede im Ausmaß der Digitalisierung, nach denen sich sechs Gruppen (Archetypen) digitaler Unternehmen unterscheiden lassen: von solchen, die die digitale Transformation schon sehr weit getrieben haben (digitale Champions) bis zu denen, die die Chancen der Digitalisierung noch fast gar nicht nutzen (digitale Nachzügler). Die Befragung unterstützt die in Abb. 1.1 genannten Ergebnisse und differenziert sie gleichzeitig deutlich aus. Bei den meisten deutschen Unternehmen ist aber entweder die strategische oder/und die operative Ausrichtung auf die Digitalisierung weder so stark gediehen wie bei den Champions noch so wenig ausgeprägt wie bei den Nachzüglern. Diese Ergebnisse sind über Branchen und Regionen weitgehend ähnlich und bieten Ansatzpunkte für Wege in die Digitalisierung, die im nächsten Kapitel aufgezeigt werden sollen. 


\section{Anmerkungen zu Kapitel 5}

1. Vgl. z. B. Meffert, Klein (2013, S. 38 f.).

2. Vgl. zur Indexbildung Bortz, Lienert (2008) oder Goldstein, Eley (2014).

3. Vgl. Grant, Verona (2015).

4. Vgl. z. B. Roland Berger (2015).

5. Vgl. Köhler, Wollschläger (2014, S. 76).

6. Vgl. Sedran, Gissler (2015, S. 1 und 3).

7. Vgl. Acatech (2014)

8. Vgl. Vargo, Lusch (2004).

9. Vgl. ebd. und Lusch, Nambisan (2015).

10. Vgl. z. B. Johnson u. a. (2008 S. 60), Proff u. a. (2014), Foss, Saebi (2017).

11. Vgl. Vargo, Lusch (2004), Lusch, Nambisan (2015).

12. Vgl. z. B. Anderson, Tushman (1990), Bucherer u. a. (2012), Abdelkafi u. a. (2013), Frankenberger u. a. (2013).

13. Vgl. Bucherer (2012), Abdelkafi u. a. (2013), Frankenberger u. a. (2013).

14. So das Ergebnis einer statistischen Poweranalyse.

15. Vgl. Foss, Saebi (2017) und Jacobides, MacDuffie (2013).

16. Vgl. dazu z. B. Meffert, Klein (2013).

17. In einer Vorstudie konnten vier Archetypen unterschieden werden und auch in der in erwähnten Studie wurden vier Typen unterschieden. Da in der Hauptuntersuchung die Fallzahl mit 160 Unternehmen größer war, konnten insgesamt sechs Cluster unterschieden werden - eine noch stärkere Ausdifferenzierung brachte keine zusätzlichen Erkenntnisse (vgl. dazu Backhaus u. a. 2016, Kap. 16).

18. Um eine Vergleichbarkeit zu erreichen, wurde für die globale Befragung die gleiche Struktur der Ar-chetypen wie in Deutschland (vgl. Abb. 5.10) angenommen. Ein Vergleich der verschiedenen Cluster-analysen zeigte, dass der dabei auftretende Fehler gering ist. Aussagen in diesem Kapitel und auch in Abschn. 5.5 sind dennoch nur als Tendenzaussagen aufzufassen. 
Open Access Dieses Kapitel wird unter der Creative Commons Namensnennung 4.0 International Lizenz (http://creativecommons.org/licenses/by/4.0/deed.de) veröffentlicht, welche die Nutzung, Vervielfältigung, Bearbeitung, Verbreitung und Wiedergabe in jeglichem Medium und Format erlaubt, sofern Sie den/die ursprünglichen Autor(en) und die Quelle ordnungsgemäß nennen, einen Link zur Creative Commons Lizenz beifügen und angeben, ob Änderungen vorgenommen wurden.

Die in diesem Kapitel enthaltenen Bilder und sonstiges Drittmaterial unterliegen ebenfalls der genannten Creative Commons Lizenz, sofern sich aus der Abbildungslegende nichts anderes ergibt. Sofern das betreffende Material nicht unter der genannten Creative Commons Lizenz steht und die betreffende Handlung nicht nach gesetzlichen Vorschriften erlaubt ist, ist für die oben aufgeführten Weiterverwendungen des Materials die Einwilligung des jeweiligen Rechteinhabers einzuholen.

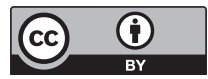

